\title{
VSOP: the variable star one-shot project ${ }^{\star}$ I. Project presentation and first data release
}

\author{
T. H. Dall ${ }^{1}$, C. Foellmi ${ }^{2}$, J. Pritchard ${ }^{3}$, G. Lo Curto ${ }^{4}$, C. Allende Prieto ${ }^{5}$, H. Bruntt ${ }^{6}$, P. J. Amado ${ }^{7}$, \\ T. Arentoft ${ }^{8}$, M. Baes ${ }^{9}$, E. Depagne ${ }^{4,10}$, M. Fernandez ${ }^{11}$, V. Ivanov ${ }^{4}$, L. Koesterke ${ }^{5}$, L. Monaco ${ }^{4}$, K. O’Brien ${ }^{4}$, \\ L. M. Sarro ${ }^{12}$, I. Saviane ${ }^{4}$, J. Scharwächter ${ }^{4}$, L. Schmidtobreick ${ }^{4}$, O. Schütz ${ }^{4}$, A. Seifahrt ${ }^{13}$, \\ F. Selman ${ }^{4}$, M. Stefanon ${ }^{4}$, and M. Sterzik ${ }^{4}$ \\ 1 Gemini Observatory, 670 N. A’ohoku Pl., Hilo, HI 96720, USA \\ e-mail: tdall@gemini.edu \\ 2 Laboratoire d'AstrOphysique de Grenoble, 414 rue de la Piscine, 38400 Saint-Martin d'Hères, France \\ 3 European Southern Observatory, Karl Schwarzschild-Str. 2, 85748 Garching bei München, Germany \\ 4 European Southern Observatory, Alonso de Cordova 3107, Casilla 19001, Vitacura, Santiago, Chile \\ 5 McDonald Observatory and Department of Astronomy, The University of Texas, Austin, TX 78712-1083, USA \\ ${ }^{6}$ School of Physics A28, University of Sydney, 2006 NSW, Australia \\ 7 Universidad de Granada-IAA(CSIC), PO Box 3004, 18080 Granada, Spain \\ 8 Department of Physics and Astronomy, University of Aarhus, 8000 Aarhus C, Denmark \\ 9 Sterrenkundig Observatorium, Universiteit Gent, Krijgslaan 281 S9, 9000 Gent, Belgium \\ ${ }_{11}$ Pontificia Universidad Catolica de Chile, Vicuna Mackenna 4860, Santiago de Chile, Chile \\ 11 Instituto de Astrofísica de Andalucía, Camino Bajo de Huétor 50, 18008 Granada, Spain \\ 12 Department of Artificial Intelligence, ETSI Informática, Juan del Rosal 16, 28040 Madrid, Spain \\ 13 Astrophysikalisches Institut und Universitäts-Sternwarte Jena, Schillergässchen 2, 07745 Jena, Germany
}

Received 29 March 2007 / Accepted 28 May 2007

\section{ABSTRACT}

\begin{abstract}
Context. About 500 new variable stars enter the General Catalogue of Variable Stars (GCVS) every year. Most of them however lack spectroscopic observations, which remains critical for a correct assignement of the variability type and for the understanding of the object.

Aims. The Variable Star One-shot Project (VSOP) is aimed at (1) providing the variability type and spectral type of all unstudied variable stars, (2) process, publish, and make the data available as automatically as possible, and (3) generate serendipitous discoveries. This first paper describes the project itself, the acquisition of the data, the dataflow, the spectroscopic analysis and the on-line availability of the fully calibrated and reduced data. We also present the results on the 221 stars observed during the first semester of the project.

Methods. We used the high-resolution echelle spectrographs HARPS and FEROS in the ESO La Silla Observatory (Chile) to survey known variable stars. Once reduced by the dedicated pipelines, the radial velocities are determined from cross correlation with synthetic template spectra, and the spectral types are determined by an automatic minimum distance matching to synthetic spectra, with traditional manual spectral typing cross-checks. The variability types are determined by manually evaluating the available light curves and the spectroscopy. In the future, a new automatic classifier, currently being developed by members of the VSOP team, based on these spectroscopic data and on the photometric classifier developed for the COROT and Gaia space missions, will be used.

Results. We confirm or revise spectral types of 221 variable stars from the GCVS. We identify 26 previously unknown multiple systems, among them several visual binaries with spectroscopic binary individual components. We present new individual results for the multiple systems V349 Vel and BC Gru, for the composite spectrum star V4385 Sgr, for the T Tauri star V1045 Sco, and for DM Boo which we re-classify as a BY Draconis variable. The complete data release can be accessed via the VSOP web site.
\end{abstract}

Key words. stars: variables: general - stars: fundamental parameters - methods: observational astronomical data bases: miscellaneous

\section{Introduction}

There are more than 38000 known variable stars listed in the latest edition of the General Catalog of Variable Stars (GCVS; Kholopov et al. 1998). Almost 4000 of these have no spectral type assigned and nearly 2000 are listed with an uncertain variability type, often because of lack of spectroscopic characterisation. The rate of inclusion of new variables is currently

^ Based on data obtained at the La Silla Observatory, European Southern Observatory, under program ID 077.D-0085. around 500 per year; actually 1700 between the Namelists 77 (Kazarovets et al. 2003) and 78 (Kazarovets et al. 2006). The true number of new variables is higher though, and this incompleteness of the GCVS will likely increase in the coming decade due to the large surveys that will be performed with both ground-based and space-based telescopes. About half of the newly identified GCVS variables have unknown variability type and most of them have no published spectral type. Moreover, many (even "firm") variables have disagreeing designations between different authors and even between different catalogs, 
e.g. there are frequent disagreements between the SIMBAD database and GCVS. In addition, binarity is rarely detectable unquestionably by photometric data alone. Finally, many designations are taken at face value without questioning the reliability. This unreliability is a major obstruction to many individual studies, and would often require only one "snapshot" spectrum to achieve a major improvement. Of course, single shot spectra would not always be able to reveal binarity or transient phenomena.

Recent examples of the misidentification of variables, where the designation was based solely on photometric light curve appearance, and subsequently corrected by taking one single snapshot spectrum, include:

FH Leo, that was long thought to be the only known cataclysmic variable $(\mathrm{CV})$ to form part of a binary system, being designated as a nova-like variable by Kazarovets et al. (2003) based on an outburst observed by the Hipparcos satellite. Highresolution FEROS spectroscopy allowed us to refute the classification and show that the stars are normal F8 and G0 dwarfs (Dall et al. 2005), and that the outburst cannot possibly be due to an accretion disk, but rather to a superflare or to erroneous Hipparcos measurements, or due to a CV "hidden" in the light of the two normal stars (Vogt 2006).

XY Pic was included in a study of statistical properties of W UMa type variables (active, very fast rotating contact binaries) by Selam (2004), who concluded that it was among the most active stars of the sample, based on a fit of its Hipparcos light curve, using synthetic light curves based on physical parameters. FEROS spectra of XY Pic allowed us to show that the star is a rather slowly rotating F3 giant, with no measurable chromospheric activity (Dall 2005), and is likely a $\delta$ Scuti pulsator. This example shows, that even a high-quality light curve analysis can result in wrong conclusions about the nature of an object without spectroscopic confirmation.

TV Ret was long thought to be a CV due to an outburst observed photometrically in 1977. A single low resolution spectrum, revealed the object to be a compact emission line galaxy at $z \sim 0.1$, possibly hosting an extremely bright supernova as the cause of the outburst (Schmidtobreick et al. 2007).

The above examples illustrate the need for snapshot spectra, and shows that the vast collection of poorly studied variable stars contains many errors in terms of variability type designation, which may in many cases "cover up" some potentially interesting physical phenomena under a wrong and seemingly dull label. In this paper we describe a new large project, the Variable Stars One-shot Project (VSOP), undertaken to provide the required "snapshots". We present the motivation and scope of VSOP in Sect. 2, the instrumentation and data handling in Sect. 3, and present results from the first observing semester from the European Southern Observatory's La Silla site in Sect. 4, listing the revised spectral and variability types for 221 stars. The results and the reduced data are freely accessible from our website. We conclude the paper with plans for the future of VSOP in Sect. 5, where we also address the problem of automatic variability classification.

\section{The project}

Motivated by the situation outlined above, the goals of VSOP are:

1. to obtain the first spectroscopy of all unstudied variable stars, revising spectral and variability types;
2. to process, publish, and make the data available as automatically as possible, facilitating additional science;

3. to generate serendipitous discoveries that will fuel future research.

In addition, due to its beginnings as a proposal for an observatory project, VSOP has been designed with a view to both science and observatory efficiency.

\subsection{Science efficiency}

A stellar spectrum is a rich source of information. Often, however, only certain aspects of the object under study is of interest to the scientist conducting the study. One main goal of VSOP is to revise spectral and variability types, but there are certainly many other scientifically interesting studies one could perform using our data. We choose, rather than to sit on the data until we may find time for additional studies, to make the data easily available to the general community, in the hope that somebody else will be able to do additional science with the data. This way, the science output is maximised.

Another aspect that contributes to the science efficiency, is serendipity. The VSOP observations are targeting poorly studied variable stars, many of which are exhibiting poorly studied phenomena. We thus expect to obtain by chance ${ }^{1}$ data that either merit follow-up in-depth studies, or sheds light on some hitherto obscured phenomenon. Much of this work may naturally be done by groups not affiliated with VSOP.

\subsection{Observatory efficiency}

VSOP was originally conceived as an ESO observatory project, aimed at providing observations with loose weather and pointing constraints, with the aim of increasing observing efficiency during periods when other programmes with stronger constraints on airmass, seeing, and/or transparency cannot be carried out. Given the all-sky coverage, the loose constraints, and the large scope of the project, VSOP is an excellent example of a perfect filler programme, which will be extended to other observatories in the near future.

\subsection{VSOP vs. other surveys}

The most extensive spectroscopic survey to date, is the Sloan Digital Sky survey (SDSS; York et al. 2000). As of the fifth data release, the SDSS provides more than 800000 spectra of galaxies, quasars and stars over nearly 7000 square degrees, mostly in the Northern hemisphere. The spectroscopy is carried out with multi-object fiber spectrographs, providing spectral resolution of $R=2000$ and spectral coverage from 3900-9100 $\AA$. The fiber aperture on the sky is $3^{\prime \prime}$, so some contamination from fainter nearby objects is possible.

We cross-correlated the GCVS with the SDSS spectroscopic list to estimate the possible contribution of the SDSS towards accurate spectroscopic classification of the variable stars. The overlap consists of 80 objects (less than $0.3 \%$ of the GCVS) and given the degree of SDSS completeness we expect some additional objects, on order of 10 , leading us to conclude that less than $0.5 \%$ of the GCVS have been covered by SDSS. The low number of stars in common is a consequence both of the faint brightness limit of the SDSS $(g>14)$, and of the science goals of the SDSS, which dictated selection for spectroscopic follow

\footnotetext{
1 "Chance favors the prepared mind" - L. Pasteur.
} 
up primarily for the extragalactic targets, obtaining spectra of stars only if some fibers remained unused.

However, spectra of a significant number of stars will be obtained under the Sloan Extension for Galactic Understanding and Exploration (SEGUE; Newberg \& Sloan Digital Sky Survey Collaboration 2003; Rockosi 2006) which plans to obtain spectra of 240000 Milky Way stars over 3500 square degrees with the same spectrograph. The goal of this survey is to provide radial velocities and metallicities with typical accuracies of $10 \mathrm{~km} \mathrm{~s}^{-1}$ and 0.3 dex respectively. SEGUE would thus complement VSOP. However, VSOP provides superior spectral resolution and $S / N$ at any given magnitude, since we use $2-4 \mathrm{~m}$-class telescopes. Plus, VSOP is already producing and releasing data.

Another large survey is the RAdial Velocity Experiment (RAVE; Steinmetz et al. 2006), aimed at kinematic studies of the local Milky Way environment. While this survey targets a large number of stars (24748 in the first data release), the spectral coverage is limited to the IR Ca triplet region, and only at a moderate resolution of 7500 . Thus, RAVE is likely not very useful as a general classification and discovery study.

Of existing surveys, the GAUDI (Solano et al. 2005) is the one most similar to VSOP. GAUDI is a photometric and spectroscopic database of objects that may be observed by the COROT mission, covering all targets down to $V=9.5$ inside the COROT accessibility zone - an area on the sky of $10^{\circ}$ radius. While GAUDI covers a small area of the sky, looking for stellar variability in all available objects, VSOP is targeting known variables all over the sky. Thus, while complimentary, our scope is different.

Furthermore, a number of spectroscopic surveys have been performed in recent years, targeting the variability of individual types or classes of stars. Examples include surveys for $\beta$ Cep stars (Telting et al. 2006), Hipparcos-selected O-B supergiants (Lefever et al. 2007), $\gamma$ Dor stars (de Cat et al. 2006), and studies of Ap star oscillations (Kurtz et al. 2006). While these are all high-resolution spectroscopic studies, they target a limited subset of stellar types, while the scope of VSOP is all of stellar variability, spanning the complete HR diagram, including all phenomena. In this respect, VSOP is a unique project, and it is our hope that VSOP will also turn out to be a unique resource for researchers of any stellar variability phenomena.

\section{Observations and data handling}

We present here the results of the first semester of VSOP observations, collected between April and October 2006 with the two high-resolution Echelle spectrographs FEROS and HARPS, of the ESO La Silla Observatory in Chile.

\subsection{Target selection}

Our target list is constructed based on a magnitude limited sample of spectroscopically unstudied southern variables drawn from the GCVS composed of stars with unknown or uncertain variability types (designations ending in ":"), in addition to the irregular or "unsolved" variables (GCVS types *, I, L, S and subclasses). This was complemented with stars having disagreeing designations according to SIMBAD, or according to recent literature. Stars were chosen to have a magnitude generally brighter than $m_{v}=10$ to be easily observable with high-resolution spectrographs on middle-sized telescopes.

The 221 stars reported here, belong to the bright end of the unstudied variables of the GCVS, which is now $\sim 40 \%$ complete (i.e., having reliable, wide wavelength coverage spectroscopy) down to $m_{v}=10$. The rate of discovery of new variables have been relatively constant in recent years, and is very low at the bright end of the distribution. Assuming similar number of observations for the coming semesters, we can expect to complete the bright end of the unstudied variables of the Southern hemisphere in 1-2 additional observing seasons. Going to fainter magnitudes, the completeness decreases rapidly, reaching a plateau of around 20-25\% fainter than $m_{v}=13$, not including as yet unrecognized variables.

\subsection{FEROS}

FEROS (Fibre-fed Extended Range Optical Spectrograph, Kaufer et al. 1999), is ESO's high resolution, high efficiency versatile spectrograph. It is a bench-mounted, thermally controlled, prism-cross-dispersed Échelle spectrograph. It provides in a single spectrogram spread over 39 orders almost complete ${ }^{2}$ spectral coverage from $\sim 350-920 \mathrm{~nm}$ at a resolving power of 48000 .

The spectrograph is fed by two fibres providing simultaneous spectra of object plus, in the case of VSOP observations, an empty sky region for background subtraction. The fibres are illuminated via apertures of $2.0^{\prime \prime}$ on the sky separated by $2.9^{\prime}$. A dedicated pipeline implemented as a MIDAS context provides, in almost real-time, extracted 1-dimensional, wavelength calibrated spectra.

FEROS Period-77 VSOP observations have been obtained with exposure times ranging from $180 \mathrm{~s}$ to $1200 \mathrm{~s}$. Given the relaxed observing constraints, signal-to-noise $(S / N)$ ranges from $\sim 10$ to $\sim 370$ at $V$. The standard calibration plan, which provides bias, flat-field, wavelength calibration and spectrophotometric standard star observations, has been used for this programme.

\subsection{HARPS}

HARPS (High Accuracy Radial velocity Planet Searcher; Mayor et al. 2003) is the ESO instrument dedicated to extrasolar planet searches through the radial velocity method. Moreover, it has proved to be very efficient as well as a general purpose high resolution spectrograph. HARPS is a fibre fed, Échelle crossdispersed spectrograph, achieving a resolution of 110000 , while covering the spectral range from $390 \mathrm{~nm}$ to $690 \mathrm{~nm}$ in $72 \mathrm{spec}-$ troscopic orders. The spectrograph is kept under vacuum and under strict temperature control to increase stability. The light is fed to the spectrograph through a fibre with a $1^{\prime \prime}$ aperture on the sky. Like FEROS, the fibre link includes two fibres, one for the scientific target and the other for sky subtraction. The efficiency of the spectrograph peaks at $\sim 8 \%$ (Blaze maximum) at $520 \mathrm{~nm}$ and is quite flat between $450 \mathrm{~nm}$ and $690 \mathrm{~nm}$.

The standard calibration set executed prior to each observing night included bias, flat-fields and wavelength calibration. The HARPS data are automatically processed upon acquisition by a dedicated pipeline developed by the HARPS consortium and which provides bias subtraction, order localization, flat fielding, cosmics filtering, order extraction (using the Horne technique, Horne 1986, assigning lower weights to the pixels away from the peak in the spatial profile at any given wavelength) and radial velocity determination through cross correlation of each spectral order with a predefined stellar mask (synthetic spectrum).

\footnotetext{
2 The two spectral ranges $853.4-854.1 \mathrm{~nm}$ and $886.2-887.5 \mathrm{~nm}$ are lost due to non overlap of the spectral orders.
} 
HARPS Period-77 VSOP observations have been obtained with exposure times ranging from $90 \mathrm{~s}$ to $1200 \mathrm{~s}$, resulting in $S / N$ between 30 and 150 , averaging to $\sim 100$ at $550 \mathrm{~nm}$.

\subsection{The VSOP wiki database and data policy}

All the data and basic information about the stars are stored in a wiki-wiki website located at http://vsop.sc.eso.org, from where the reduced data of this First Data Release can be freely accessed. We expect to make incoming data freely available through subsequent data releases, with only a few months delay to allow for our inital data analysis. Research work benefiting from VSOP data should reference this paper, and include the following acknowledgement:

Based on data provided by the VSOP collaboration, through the VSOP wiki database operated at ESO Chile and ESO Garching.

For the organization of information, we have chosen the MediaWiki software, developed for the open and free on-line encyclopedia Wikipedia. This ensure a reliable and extendable website where all VSOP members can contribute easily from their own daily workplace. This is of growing importance given the distribution of VSOP members around the world, as evidenced by the list of affiliations for this paper.

The MediaWiki software is based on the article/discussion wiki philosophy, which means that to each article page there is an associated discussion page. For VSOP we have extended the software to make the discussion pages restricted to VSOP members only, while the article pages are reserved for already published results, freely accessible to anybody. Thus, each star has a dedicated article page, where basic informations (coordinates, magnitude, link to SIMBAD, finding charts, old variability and spectral types - when available) are provided. Also, the observation details are described as well as the analysis, its results, a list of references, catalogues and download links to plots of the spectra as well as to all the reduced data products: Cross-Correlation Function (CCF) and wavelength calibrated one-dimensional spectrum, all of which are publicly available.

Finally, the MediaWiki software allows the wiki website to be scriptable. We have thus developed a VSOP-dedicated software module written in Python which makes the development of scripts dedicated to VSOP pages much easier. These robot scripts can then update a large amount of repetitive information, or collect the results of given subcategories of stars. Table 2 of this paper, for instance, is automatically produced by one of these scripts.

\subsection{The VSOP dataflow}

The VSOP dataflow comprises a collection of different steps that are developed in order to make it as automatic as possible. The following is an outline:

1. a list of targets is build, as described in Sect. 3.1;

2. when observing time is granted, we automatically generate one wiki-page per target;

3. observations for each target are defined and submitted to the observatory database;

4. observations are carried out through the observing semester, and data is automatically reduced by the instrument pipelines (Sects. 3.2 and 3.3);
5. raw and reduced data are automatically transferred to the VSOP machine at ESO Vitacura (Sect. 3.4) and the wiki star page is updated;

6. automatically generated plots of spectra and CCF are included in the star page (Sect. 4.1);

7. VSOP members receive an email alert that new VSOP observations have been obtained;

8. analysis is undertaken, and the wiki-pages are updated if needed.

The dataflow has proved very smooth and efficient throughout the first observing season. For the following seasons, we have in addition implemented automatic spectral analysis (Sect. 4.2). However, in order to make it completely automatic, one needs to develop an automatic variability classifier, incorporating both spectroscopy and the available photometry. As already mentioned, this is an area where VSOP will play an active future role (Sect. 5).

\section{Results}

Table 2 lists the 221 stars observed during ESO Period 77: 90 of these were observed with HARPS, 131 with FEROS.

\subsection{Radial velocities and binary status}

Radial velocities (RVs) are computed via the Cross Correlation Function (CCF) method, in which a template synthetic spectrum with box shaped lines is correlated with the star spectrum to measure the Doppler line shift. Details can be found in Baranne et al. (1996). The HARPS online pipeline provides accurate radial velocities $\left(\approx 1 \mathrm{~m} \mathrm{~s}^{-1}\right)$ for slowly rotating late-F, G, K and early M dwarfs. Similarly, FEROS demonstrated a RV accuracy of $\approx 20 \mathrm{~m} \mathrm{~s}^{-1}$, the difference with HARPS being mainly due to the mechanical stability of the latter and the different choice of light injection in the two instruments. Correlation with mid to late $\mathrm{M}$ type stars is problematic due to the amount of wide molecular features in their spectra and to the paucity of narrow metal lines. Earlier type stars often have higher rotational velocities and weaker metallic lines, limiting the accuracy of the radial velocity determination but, at least for stars with metal lines, still allowing the computation of a $\mathrm{CCF}$ and therefore the estimation of the RV. In the presence of only few metal lines in the early type stars, the correlation with the G2 template (the "earliest" available for HARPS) will return a $\mathrm{CCF}$ with a small contrast (few \%). Due to the scarcity of narrow metal lines, early-type templates will necessarily include strong lines which naturally suffer from asymmetries, which in turn will lower the RV precision. For the time being, the RV of earlier spectral types is estimated by automatic fitting of the core of $\mathrm{H} \beta$ with a second-order polynomial as part of the VASP analysis (cf. Sect. 4.2). While techniques such as the cross correlation in Fourier space with template spectra obtained from observations allows relative accuracies of $\approx 10 \mathrm{~m} \mathrm{~s}^{-1}$ in RVs on early type stars (Galland et al. 2005), we use the faster CCF method, as such an accurate RV determination is not our primary concern.

We designate SB2 and SB3 binarity status from the presence of multiple peaks in the CCF, or via a careful analysis of the spectrum, identifying spectral features belonging to stars of different spectral types. The latter case is when the stars have widely different spectral types, and the CCF mask only "sees" one of the components (see e.g. V4385 Sgr, Sect. 4.3.3). Since we have only one epoch, the SB1 designation is not used. 
Table 1. A sample of results from the automatic analyses. We list the names, HD numbers and spectral types from the manual spectral typing. We give the $T_{\text {eff }}, \log g$ and $[\mathrm{Fe} / \mathrm{H}]$ and estimated uncertainties determined by VASP and VWA. $v \sin i$ values are calculated with VWA and have uncertainties of 10-20\%. The first four targets are not VSOP targets, but high S/N HARPS spectra taken from Dall et al. (2006), which we have used to calibrate our tools. Full detailed results for all VSOP stars can be found online (see text).

\begin{tabular}{lrlr|lcc|ccc}
\hline \hline Star & & Spectral & $v \sin i$ & \multicolumn{3}{c|}{ VASP } & \multicolumn{3}{c}{ VWA } \\
& HD & type & {$\left[\mathrm{km} \mathrm{s}^{-1}\right]$} & $T_{\text {eff }}[\mathrm{K}]$ & $\log g$ & {$[\mathrm{Fe} / \mathrm{H}]$} & $T_{\text {eff }}[\mathrm{K}]$ & $\log g$ & {$[\mathrm{Fe} / \mathrm{H}]$} \\
\hline$\alpha$ Hor & 26967 & K1III & 2 & $4675(143)$ & $2.8(4)$ & $+0.22(13)$ & $4550(80)$ & $2.2(2)$ & $-0.02(5)$ \\
$\tau$ Cet & 10700 & G8V & 3 & $5292(157)$ & $4.6(4)$ & $-0.45(15)$ & $5320(50)$ & $4.5(1)$ & $-0.47(5)$ \\
Sun & - & G2V & 2 & $5842(168)$ & $4.5(4)$ & $-0.16(16)$ & $5810(40)$ & $4.5(1)$ & $-0.07(3)$ \\
$\gamma$ Ser & 142860 & F6IV & 11 & $6322(191)$ & $4.1(7)$ & $-0.18(20)$ & $6250(80)$ & $4.1(1)$ & $-0.24(5)$ \\
\hline ZZ Pyx & - & K3V & 5 & $4228(223)$ & $1.4(1)$ & $-0.31(13)$ & $3900(150)$ & $1.4(3)$ & $-1.3(2)$ \\
V349 Vel & 91021 & F2+? & 16 & $7314(241)$ & $5.2(9)$ & $-0.89(54)$ & $7200(150)$ & $4.0(2)$ & $-0.43(5)$ \\
LP Vir & 115466 & F1IV & 40 & $7031(194)$ & $3.4(8)$ & $-0.32(29)$ & $7030(80)$ & $3.7(1)$ & $-0.13(5)$ \\
V976 Cen & 118551 & F0III & 40 & $7048(203)$ & $2.0(7)$ & $-0.16(23)$ & $8400(100)$ & $2.4(2)$ & $-0.78(5)$ \\
PP Hya & 87130 & A5V & 80 & $7924(219)$ & $4.0(8)$ & $-1.35(73)$ & - & - & - \\
\hline
\end{tabular}

Out of the 221 observed targets, we identify 22 new SB2 binaries, several of these as components of wide visual binaries. In addition we find four new SB3 binaries. However, the binarity of most of our targets remains undetermined due to our singleepoch approach. For many of the stars we could not compute a $\mathrm{CCF}$, due to the difficulty to build reliable templates for such peculiar objects. We postpone accurate determination of the binary status of such stars to a later work.

\subsection{Spectral classification}

The first set of atmospheric parameters $\left(T_{\text {eff }}, \log g\right.$, $\left.[\mathrm{Fe} / \mathrm{H}]\right)$ listed in Table 1 have been obtained automatically by comparison of the observed spectra with theoretical spectra in a region around $\mathrm{H} \beta$ (486 nm). This spectral window can be used to uniquely constrain the atmospheric parameters for stars with spectral types A to K (Allende Prieto 2003). This automated software (VASP: VSOP Automatic Stellar Parameters) and the synthetic spectra are very similar to those used for the STELLA robotic telescopes (Strassmeier et al. 2007, in preparation).

The search for the optimal solution is carried out using the Nelder-Mead algorithm and third order interpolation in a grid of synthetic spectra based on Kurucz (2006) model atmospheres and modern line and continuous opacities ${ }^{3}$ (Allende Prieto et al. $2003 \mathrm{~b}, \mathrm{a})$. The grid currently in use covers $4500<T_{\text {eff }}<7500 \mathrm{~K}$. To overcome problems with fast rotation, the grid has been constructed with a resolution of $38 \mathrm{~km} \mathrm{~s}^{-1}$, corresponding to a spectral resolving power of 7700 . For faster rotational velocities, the accuracy of the fits decrease significantly as rotation increases.

The solar reference abundances are from the photospheric values compiled by Asplund et al. (2005). Known spectroscopic binaries (cf. Sect. 4.1), as well as stars clearly outside the grid boundaries, are not run through VASP.

Future upgrades to VASP will include wider temperature range grids, as currently only about one third of our targets fall within the limits of the grid. Other upgrades will be parameter estimation using other spectral intervals besides $\mathrm{H} \beta$, and the ability to handle rotational broadening.

For comparison, we have included results from a classical abundance analysis obtained with the $\mathrm{VWA}^{4}$ package (Bruntt et al. 2004). VWA works with the original (full resolution)

\footnotetext{
${ }^{3}$ Also Kurucz (1993) and http://kurucz.harvard.edu/, the odfnew grid.

${ }^{4}$ VWA is available here: http://www .hans.bruntt.dk/vwa/
}

spectra and determines the abundance of each individual line. It relies on the lines of $\mathrm{Fe}, \mathrm{Cr}$ and $\mathrm{Ti}$ to automatically adjust the microturbulence, $T_{\text {eff }}$ and $\log g$ of the applied atmospheric models (Heiter et al. 2002). VWA is a semi-automatic procedure and to obtain optimal results the user needs to make (1) a careful correction of the continuum and (2) inspect the fit of individual lines. The abundances found with VWA are based on corrected $\log g f$ values, which are derived from the HARPS spectrum of the Sun. We did not analyse PP Hya due to its high $v \sin i$, which is known to cause problems for VWA's automatic procedures. While VWA seems to produce more robust results, the process involves a lot of manual intervention and is not at this point suited for an automated analysis.

We have in addition performed manual spectral classifications, by comparison with standard stars of the MK spectral classification as defined by Morgan et al. (1978) and Keenan \& McNeil (1976). The practical comparison has been done with the help of the Digital Spectral Classification Atlas by Gray ${ }^{5}$, using high resolution spectra of spectral standards obtained with HARPS, FEROS and UVES. Whenever the emission cores of the Ca II H\&K lines have been present, we have determined the luminosity type from the Wilson-Bappu effect (Wilson \& Vainu Bappu 1957), using the calibration by Pace et al. (2003). Since many older classifications are done in this way, it is instructive to investigate the differences between this traditional human skill driven task, and a modern automatic classification.

We have identified several causes for problems in the spectral classifications, the most common one associated with binarity. Spectroscopic and very close visual binaries (separations $<1.5^{\prime \prime}$ ) often show multiple peaks in the CCF, and are thus easy to filter out of the VASP analysis. One such example is V349 Vel (Sect. 4.3.1). We also found that early-type and very metalweak stars limits the precision of the VASP fit, and of course influences also the manual classification. In many cases, apparent low metallicity may be due to a binary companion contributing light to the spectrum, causing the metallic lines to appear weaker. One clear example of this can be seen in V4385 Sgr (Sect. 4.3.3). In a few cases we have found disagreement between the VASP-computed $\log g$ and the absolute magnitude derived from the Wilson-Bappu relation, in most cases caused by being near the lower temperature limit of the grid.

\footnotetext{
5 http://nedwww.ipac. caltech.edu/level5/Gray/ frames.html
} 
Table 2. The VSOP stars of the first observing season.

\begin{tabular}{|c|c|c|c|c|c|c|c|c|}
\hline \multicolumn{9}{|c|}{ Properties of the stars in our sample } \\
\hline GCVS name & A.k.a. & Date & Ins. & $m_{V}$ & Var. & Spec. & Bin. & Notes \\
\hline AI Pyx & GSC 07145-02569 & 2006-04-01 & $\mathrm{H}$ & 6.23 & IA & B3V & $?$ & Old Spec $^{6}:$ B4V \\
\hline AL Leo & GSC 01415-01312 & 2006-04-01 & $\mathrm{H}$ & 9.92 & EA/D & F5V & $\mathrm{Y}$ & OS:F5 \\
\hline AN Pyx & GSC 06039-01085 & 2006-04-01 & $\mathrm{H}$ & 8.25 & $\mathrm{ACV}$ & A2V-IV & ? & OS: A0V \\
\hline AO Ant & GSC 07179-02338 & 2006-06-11 & $\mathrm{F}$ & 8.56 & ? & M4III & VIS & OS: M0 \\
\hline AQ Ant & GSC 07187-00952 & 2006-06-13 & $\mathrm{F}$ & 9.02 & ? & M3III & ? & OS: K5 \\
\hline AR Ant & GSC 07708-00615 & 2006-04-09 & $\mathrm{F}$ & 9.3 & LB & M4III & ? & OS: M \\
\hline AY Ant & GSC 07727-00703 & 2006-06-12 & $\mathrm{F}$ & 9.84 & ? & K0e & ? & OS: Kp \\
\hline BC Gru & & 2006-08-09 & $\mathrm{F}$ & 9.9 & EW & $\mathrm{K} 0+\mathrm{K} 0+\mathrm{K}$ & SB3 & OS: ?, Old Bin ${ }^{7}:$ Y \\
\hline BH Cap & GSC 05762-02497 & 2006-04-02 & $\mathrm{H}$ & 8.03 & $\mathrm{~EB}$ & $\mathrm{~F} 2 \mathrm{IV}+\mathrm{F} 2 ?$ & SB2 & OS: F0, OB: Y \\
\hline BK Cap & GSC 06338-00216 & $2006-05-12$ & $\mathrm{~F}$ & 8.78 & LB & M1III & ? & OS: M1/M2III: \\
\hline BM Scl & GSC $07512-00800$ & 2006-08-07 & $\mathrm{F}$ & 8.28 & LB & M1III & ? & OS: M2III \\
\hline BP Psc & GSC 05244-00148 & $2006-06-29$ & $\mathrm{H}$ & 9.04 & IT & G2IVe & ? & OS:? \\
\hline BP Scl & GSC 07512-00221 & 2006-08-07 & $\mathrm{F}$ & 8.07 & LB & K7III & ? & OS: K5III \\
\hline BQ Scl & GSC 07507-01012 & 2006-06-16 & $\mathrm{F}$ & 8.88 & ? & K5III & ? & OS: MOIII: \\
\hline BS Pav & & 2006-04-02 & $\mathrm{H}$ & 9.8 & IS & M6 & ? & OS:? \\
\hline BW CMi & GSC 00206-01099 & 2006-04-18 & $\mathrm{F}$ & 9.02 & LB & K8V & $?$ & OS: M0 \\
\hline BW Pyx & GSC 06590-00111 & 2006-04-18 & $\mathrm{F}$ & 9.93 & SR & M2III & ? & OS: M \\
\hline BZ Ind & GSC 09324-00534 & $2006-08-10$ & $\mathrm{~F}$ & 8.72 & LB & M4III & ? & OS: M3III \\
\hline CL Phe & GSC $08457-00425$ & 2006-06-09 & $\mathrm{H}$ & 9.7 & BY & K2IV-V & ? & OS: K1V \\
\hline CP Cir & GSC 09019-01463 & 2006-04-02 & $\mathrm{H}$ & 7.51 & GCAS & B5V & ? & OS: B5IV \\
\hline CS Gru & GSC 07996-00969 & $2006-05-21$ & $\mathrm{H}$ & 9.45 & BY & G8V & $\mathrm{Y}$ & OS: G8/K0V, OB:? \\
\hline CX Gru & GSC 07997-00367 & 2006-06-09 & $\mathrm{H}$ & 6.66 & ELL & $\mathrm{F} 8 \mathrm{~V}+\mathrm{F} 8 \mathrm{~V}$ & SB2 & OS: F7V, OB: VIS \\
\hline DG Oct & GSC 09469-00374 & $2006-06-13$ & $\mathrm{~F}$ & 8.90 & $?$ & M3III & $?$ & OS: M3III: \\
\hline DG Psc & GSC 00575-00094 & 2006-08-09 & $\mathrm{F}$ & 8.74 & LB & M4III & ? & OS: M \\
\hline DH Psc & GSC 00569-00289 & 2006-08-09 & $\mathrm{F}$ & 8.69 & LB & M3III & ? & OS: K5 \\
\hline DI Psc & GSC 00575-00918 & 2006-08-08 & $\mathrm{F}$ & 7.28 & LB & G8III-IV & ? & OS: K0 \\
\hline DK Psc & GSC 00576-00284 & 2006-08-09 & $\mathrm{F}$ & 8.38 & LB & M3III & ? & OS: M \\
\hline DM Boo & GSC 00903-00938 & 2006-08-12 & $\mathrm{F}$ & 8.73 & BY & G2 & $?$ & Old $\operatorname{Var}^{8}:$ IB, OS: G5 \\
\hline DR Cru & GSC $08655-01039$ & 2006-07-02 & $\mathrm{F}$ & 8.88 & BY & $\mathrm{K} 5 \mathrm{~V}+\mathrm{K}$ & SB2 & OS: K3/K4V, OB:? \\
\hline DS Cha & GSC 09418-00570 & 2006-04-13 & $\mathrm{F}$ & 8.96 & LB & M2III & $?$ & OS: M2 \\
\hline DT Cha & GSC 09415-00568 & 2006-04-21 & $\mathrm{F}$ & 8.56 & LB & K3IV & ? & OS: K3III \\
\hline DT Tuc & GSC 08839-00456 & 2006-08-09 & $\mathrm{F}$ & 9.09 & LB & M4III & ? & OS: M3III \\
\hline DW Tuc & GSC 09130-00332 & 2006-08-09 & $\mathrm{F}$ & 9.04 & LB & M2III & $?$ & OS: M1III \\
\hline DX Tuc & GSC 09130-01530 & 2006-08-08 & $\mathrm{F}$ & 9.63 & EW & $\mathrm{F} 5+\mathrm{F}$ & SB2 & OS: F7IV/V, OB: Y \\
\hline EF Aqr & GSC 05248-01030 & $2006-06-29$ & $\mathrm{H}$ & $10.3 ?$ & ELL & G0+G? & SB2 & OS: G0, OB: Y \\
\hline EO Boo & GSC 01481-00660 & 2006-09-16 & $\mathrm{F}$ & 8.45 & LB & M1III & $?$ & OS: M2III \\
\hline FQ Leo & GSC 00267-00569 & 2006-04-21 & $\mathrm{F}$ & 8.19 & LB & M2IV-III & ? & OS: K5 \\
\hline FQ Lup & GSC $07339-01070$ & 2006-09-09 & $\mathrm{F}$ & 9.5 & $\mathrm{~L}$ & M7 & ? & OS: M5/M6II: \\
\hline GK Cnc & GSC 01401-00763 & 2006-06-14 & $\mathrm{F}$ & 9.13 & ? & M4III & ? & OS: M \\
\hline GSC 00244-00434 & & 2006-04-18 & $\mathrm{F}$ & 10.4 & ? & FOIV & VIS & OS: F5 \\
\hline GSC 7831-0069 & & 2006-09-01 & $\mathrm{F}$ & 10.48 & $? ?$ & F0 & $?$ & OS:? \\
\hline GSC 9027-4849 & & 2006-06-13 & $\mathrm{F}$ & & $?$ & $\mathrm{~K} 0+\mathrm{K} ?$ & SB2 & OS: ?, OB: ? \\
\hline HD 109962 & GSC 08232-01689 & 2006-04-13 & $\mathrm{F}$ & 9.54 & $?$ & F0IV-III & $?$ & OS: F2V \\
\hline HD 117316 & GSC 09254-01886 & 2006-04-13 & $\mathrm{F}$ & 8.23 & ? & FOIII & ? & OS: F2IV \\
\hline HD 156542 & GSC 06241-00434 & 2006-09-07 & $\mathrm{F}$ & & $?$ & F3IV-III & ? & OS: F0V \\
\hline HD 89027 & GSC 04910-01309 & 2006-06-13 & $\mathrm{F}$ & & ? & F1V & ? & OS: F0 \\
\hline HD 95671 & GSC 08619-02281 & 2006-04-01 & $\mathrm{H}$ & 9.83 & $?$ & G0V & $?$ & OS: G0 \\
\hline HD 95673 & & 2006-04-21 & $\mathrm{F}$ & 9.0 & $?$ & A0V & VIS & \\
\hline HX Lib & GSC 06752-00434 & 2006-09-08 & $\mathrm{F}$ & 8.45 & LB & M1III & $?$ & OS: MOIII \\
\hline HZ Lib & GSC $05580-00356$ & 2006-09-08 & $\mathrm{F}$ & 8.02 & LB & MOIII & $?$ & OS: M \\
\hline IK Lib & GSC 06761-00434 & 2006-09-08 & $\mathrm{F}$ & 7.91 & LB & M1III & ? & \\
\hline IM Vir & GSC 04955-00912 & 2006-04-02 & $\mathrm{H}$ & 9.69 & $?$ & $\mathrm{G} 8 \mathrm{~V}+\mathrm{K} 2 \mathrm{~V}$ & SB2 & OS: G5, OB: Y \\
\hline IP Lib & GSC 06775-00038 & 2006-04-02 & $\mathrm{H}$ & 9.97 & BY & K0V & VIS & OS: G5/G6V \\
\hline IR Lup & GSC 08278-01151 & 2006-09-01 & $\mathrm{F}$ & 8.49 & LB & M2III & ? & OS: M2/M3III \\
\hline IX Lib & GSC 05604-00579 & 2006-09-08 & $\mathrm{F}$ & 8.54 & LB & M2III & $?$ & OS: M \\
\hline IX Lup & GSC 08698-00010 & 2006-06-10 & $\mathrm{F}$ & 8.32 & $?$ & M2III & ? & \\
\hline IX Vir & GSC 04931-00916 & 2006-04-21 & $\mathrm{F}$ & 8.22 & LB & M2III & $?$ & OS: M \\
\hline IY Lib & GSC 05597-00200 & 2006-09-08 & $\mathrm{F}$ & 7.9 & LB & M0III & ? & OS: K5 \\
\hline IY Lup & GSC 07316-00591 & 2006-09-08 & $\mathrm{F}$ & 8.23 & LB & M1III & ? & OS: M2III \\
\hline IY Vir & GSC 00865-00094 & 2006-04-21 & $\mathrm{F}$ & 9.43 & LB & K8III & ? & OS: M0 \\
\hline $\mathrm{KK} \operatorname{Tr} \mathrm{A}$ & & 2006-08-11 & $\mathrm{F}$ & 9.5 & $\mathrm{~L}$ & M7 & $?$ & OS: M5Ib: \\
\hline KL Lup & GSC 07822-00250 & 2006-09-09 & $\mathrm{F}$ & 8.23 & LB & MOIII & ? & OS: K5III \\
\hline KM Aqr & GSC 05802-00227 & 2006-08-10 & $\mathrm{F}$ & 8.13 & LB & M3III & ? & OS: M \\
\hline
\end{tabular}


Table 2. continued.

\begin{tabular}{|c|c|c|c|c|c|c|c|c|}
\hline \multicolumn{9}{|c|}{ Properties of the stars in our sample } \\
\hline KM Lup & GSC 07830-02867 & $2006-09-13$ & $\mathrm{~F}$ & 7.57 & LB & M2 & $?$ & OS: M2III \\
\hline KN Com & GSC 00881-00011 & 2006-06-13 & $\mathrm{F}$ & 8.81 & ? & M3III & ? & OS: M0 \\
\hline KN Lup & GSC 07313-00641 & 2006-04-02 & $\mathrm{H}$ & 9.21 & BY & G7V & SB2 & OS: G0V, OB:? \\
\hline KN Vir & GSC 04944-01258 & 2006-04-01 & $\mathrm{H}$ & 7.41 & LB & K7III & ? & OS: K5 \\
\hline KO Lup & GSC $07317-00457$ & 2006-09-08 & $\mathrm{F}$ & 6.89 & LB & M6III & ? & OS: M3/M4III \\
\hline KP Lup & GSC 08299-02622 & 2006-09-13 & $\mathrm{F}$ & 7.93 & LB & M5III & ? & OS: M4III \\
\hline KQ Aqr & GSC 06955-01363 & 2006-06-04 & $\mathrm{H}$ & 9.53 & $\mathrm{ACV}$ & $\mathrm{A} 0 \mathrm{~V}$ & ? & \\
\hline KQ Vir & GSC 04941-00938 & 2006-04-01 & $\mathrm{H}$ & 8.84 & LB & M1III & ? & OS: K5 \\
\hline KS Aqr & GSC 05228-00455 & $2006-08-10$ & $\mathrm{~F}$ & 9.42 & LB & M4III & ? & OS: M1 \\
\hline KS Mus & GSC 09230-01722 & 2006-04-13 & $\mathrm{F}$ & 8.07 & LB & M1III & ? & OS: M2III \\
\hline KX Lup & GSC $07837-01552$ & 2006-04-12 & $\mathrm{F}$ & 8.34 & LB & M1III & ? & OS: M2III \\
\hline KY Aqr & GSC $05239-01000$ & $2006-08-10$ & $\mathrm{~F}$ & 8.81 & LB & M3III & ? & OS: K5 \\
\hline LO Aqr & GSC 05811-01661 & $2006-08-10$ & $\mathrm{~F}$ & 7.44 & IB & A9 & ? & OS: F0 \\
\hline LO Mus & GSC 09000-00155 & 2006-04-02 & $\mathrm{H}$ & 8.61 & BY & $\mathrm{K} 2 \mathrm{~V}$ & ? & OS: K0V \\
\hline LP Vir & GSC 05547-01517 & 2006-04-01 & $\mathrm{H}$ & 6.92 & ELL & F1IV & $?$ & OS: F0 \\
\hline LV Нyа & GSC 07222-01221 & 2006-04-01 & $\mathrm{H}$ & 6.2 & $\mathrm{ACV}$ & A0III & $?$ & OS: A0V \\
\hline LW Vir & GSC 05545-00751 & 2006-04-09 & $\mathrm{F}$ & 9.14 & LB & K8V-IV & $?$ & OS: K5 \\
\hline MS TrA & GSC 09041-00093 & $2006-05-16$ & $\mathrm{H}$ & 8.86 & $\mathrm{ACV}$ & F2III & SB & OS: A9:IV:p, OB:? \\
\hline MS Vir & GSC 06141-00265 & 2006-09-07 & $\mathrm{F}$ & 9.4 & EW & $\mathrm{K} 0+\mathrm{K} 5$ & SB2 & OS: K0/K1III/IV, OB: Y \\
\hline MT TrA & GSC 09278-02611 & $2006-08-27$ & $\mathrm{~F}$ & 8.44 & LB & M1III & $?$ & OS: M2III \\
\hline MW Vir & GSC 06147-00662 & $2006-05-21$ & $\mathrm{~F}$ & 6.95 & $?$ & F0V & Y & OS: A5IV \\
\hline MY Vir & GSC 00320-00214 & $2006-06-13$ & $\mathrm{~F}$ & 8.30 & $?$ & M3III & $?$ & OS: M \\
\hline NN Hya & GSC 05451-00040 & $2006-06-10$ & $\mathrm{~F}$ & 6.59 & $?$ & MOIII & $?$ & OS: K5 \\
\hline NP Del & GSC 01647-00196 & 2006-05-04 & $\mathrm{H}$ & 8.89 & ELL & A3IV & $?$ & OS: A0 \\
\hline NP Hya & GSC 00229-00560 & 2006-04-01 & $\mathrm{H}$ & 7.08 & $\mathrm{ACV}$ & A0III & VIS & OS: A2 \\
\hline NQ Hya & GSC 06031-00276 & 2006-04-09 & $\mathrm{F}$ & 7.97 & LB & K7IV & $?$ & OS: M1III \\
\hline NQ Vel & GSC 07673-01114 & 2006-04-01 & $\mathrm{H}$ & 7.63 & IA & $\mathrm{B} 5 \mathrm{I}+\mathrm{B} 8 ?$ & SB2 & OS: A5, OB:? \\
\hline NR Peg & GSC 01649-00371 & $2006-05-21$ & $\mathrm{H}$ & 8.13 & BY & G5III+? & SB2 & OV: EB, OS: G0, OB: Y \\
\hline NR Vel & GSC 08158-01896 & 2006-04-01 & $\mathrm{H}$ & 7.56 & GCAS & $\mathrm{B} 2 \mathrm{e}$ & $?$ & OS: B2V:e \\
\hline NS Peg & GSC 01654-00963 & 2006-08-06 & $\mathrm{F}$ & 7.92 & LB & M3 & $?$ & OS: M \\
\hline NS Vel & GSC 07662-03038 & 2006-06-06 & $\mathrm{F}$ & 7.32 & $?$ & B6IIIe & $?$ & OS: B6III/IV \\
\hline NV Aps & GSC 09266-02415 & $2006-05-21$ & $\mathrm{~F}$ & 8.87 & $?$ & K7III & $?$ & OS: K5 \\
\hline NV Hya & GSC 05462-01496 & 2006-06-12 & $\mathrm{F}$ & 7.53 & $?$ & G8III & $?$ & OS: K0 \\
\hline NW Hya & GSC 04892-00755 & 2006-06-14 & $\mathrm{F}$ & 7.75 & $?$ & K5III & $?$ & OS: K5 \\
\hline NX Peg & GSC $00550-00385$ & 2006-08-08 & $\mathrm{F}$ & 8.1 & LB & M3III & ? & OS: M \\
\hline OP Ser & GSC 00920-00630 & $2006-06-10$ & $\mathrm{~F}$ & 8.32 & ? & K0III+? & SB2 & OS: K0, OB:? \\
\hline OQ Hya & GSC 06034-00871 & $2006-06-16$ & $\mathrm{~F}$ & 7.98 & $?$ & MOIII & $?$ & OS: M1III \\
\hline OS Peg & GSC $01122-00360$ & 2006-08-11 & $\mathrm{F}$ & 9.06 & LB & M2III & $?$ & OS: M0 \\
\hline OT Peg & GSC 01679-00700 & $2006-05-21$ & $\mathrm{H}$ & 9.89 & BY & $\mathrm{G} 8 \mathrm{IV}+\mathrm{G} 2 \mathrm{~V}$ & SB2 & OS: K0, OB:? \\
\hline OU Aps & GSC 09286-01523 & 2006-04-01 & $\mathrm{H}$ & 8.6 & $\mathrm{ACV}$ & A1IV & $?$ & OS: A0IV/V \\
\hline OU Hya & GSC 00241-01908 & 2006-06-13 & $\mathrm{F}$ & 9.60 & $?$ & M3III & $?$ & OS: M2 \\
\hline OV Aps & GSC 09443-01367 & 2006-04-01 & $\mathrm{H}$ & 8.14 & $\mathrm{ACV}$ & $\mathrm{A} 7 \mathrm{~V}+\mathrm{A} 7 \mathrm{~V}$ & SB2 & OS: A7III, OB:? \\
\hline OV Hya & GSC 06038-00531 & 2006-06-11 & $\mathrm{F}$ & 8.76 & $?$ & M4III & $?$ & OS: M3/M4III \\
\hline OX Vel & GSC 08582-02734 & 2006-04-01 & $\mathrm{H}$ & 7.6 & $\mathrm{ACV}$ & F3IV-III & $?$ & OS: A4m \\
\hline PP Нya & GSC 06054-00244 & 2006-04-01 & $\mathrm{H}$ & 6.84 & ELL & $\mathrm{A} 5 \mathrm{~V}$ & $?$ & OS: A3III \\
\hline PP Vel & GSC 08582-00358 & 2006-06-10 & $\mathrm{F}$ & 8.35 & $?$ & M1III & $?$ & OS: M2/M3 \\
\hline PQ Hya & GSC 06046-0017 & 2006-06-11 & $\mathrm{F}$ & 9.00 & $?$ & M1III & $?$ & OS: M0 \\
\hline PT Hya & GSC 05489-01121 & 2006-06-12 & $\mathrm{F}$ & 8.04 & $?$ & M2III & $?$ & \\
\hline PU Hya & GSC 05496-00657 & 2006-06-14 & $\mathrm{F}$ & 8.87 & $?$ & M4III & $?$ & OS: M3III \\
\hline PW Peg & GSC 00566-01526 & $2006-08-10$ & $\mathrm{~F}$ & 8.61 & LB & M1III & $?$ & OS: K5 \\
\hline PW Ser & GSC 00367-00383 & 2006-04-12 & $\mathrm{F}$ & 8.26 & LB & MOIII & $?$ & OS: K5 \\
\hline PW Vel & GSC 07678-02187 & 2006-06-11 & $\mathrm{F}$ & 8.52 & $?$ & M5III & $?$ & OS: M3III \\
\hline PX Peg & GSC 01695-01012 & 2006-08-10 & $\mathrm{F}$ & 8.78 & LB & MOIII & $?$ & OS: K5 \\
\hline PX Ser & GSC $00379-00580$ & 2006-04-12 & $\mathrm{F}$ & 9.33 & LB: & $\mathrm{K} 2 \mathrm{~V}+\mathrm{K} 5(?)$ & SB2 & OS: K2, OB:? \\
\hline PY Hya & GSC 05505-00401 & 2006-06-13 & $\mathrm{F}$ & 8.88 & $?$ & M2III & $?$ & \\
\hline PZ Peg & GSC 01157-00471 & $2006-08-10$ & $\mathrm{~F}$ & 9.65 & LB & K0 & $?$ & \\
\hline QQ Hya & GSC 07186-00100 & 2006-06-14 & $\mathrm{F}$ & 6.97 & $?$ & M2II-III & $?$ & OS: M2III \\
\hline QQ Ser & GSC 05683-01296 & $2006-05-24$ & $\mathrm{~F}$ & 7.66 & $?$ & M3III & $?$ & OS: M2III \\
\hline QS Peg & GSC 01158-01116 & $2006-08-10$ & $\mathrm{~F}$ & 7.96 & LB & MOIII & $?$ & OS: K5 \\
\hline QV Hya & GSC 07221-00698 & 2006-04-09 & $\mathrm{F}$ & 9.05 & LB & MOIV-III & $?$ & OS: M1III: \\
\hline RX Gru & GSC 08008-00397 & 2006-06-09 & $\mathrm{H}$ & 10.57 & EB & $\mathrm{G} 8 \mathrm{~V}+\mathrm{G} ?$ & SB2 & OS: ?, OB: Y \\
\hline SX Equ & GSC 01103-02568 & 2006-06-13 & $\mathrm{F}$ & 8.86 & ? & M1IV-III & VIS & OS: K5 \\
\hline
\end{tabular}


Table 2. continued.

\begin{tabular}{|c|c|c|c|c|c|c|c|c|}
\hline \multicolumn{9}{|c|}{ Properties of the stars in our sample } \\
\hline TV Sex & GSC 00239-01070 & $2006-06-13$ & $\mathrm{~F}$ & 8.78 & $?$ & M2III & $?$ & OS: K5 \\
\hline TW Sex & GSC 04896-01383 & 2006-06-13 & $\mathrm{F}$ & 7.93 & ? & M4III & ? & OS: M4 \\
\hline TYC 7798- 500-1 & & $2006-05-21$ & $\mathrm{~F}$ & & $?$ & F5III & $?$ & OS: ? \\
\hline UV Crv & GSC 06108-00927 & 2006-04-01 & $\mathrm{H}$ & 9.38 & BY & $\mathrm{K} 3 \mathrm{IV}+?$ & SB3 & OS: K1V, OB: VIS \\
\hline UW Crt & GSC 06090-01429 & 2006-04-09 & $\mathrm{F}$ & 8.24 & LB & K5/7V-IV & ? & OS: K5/M0III \\
\hline UW Sex & GSC 05495-00576 & 2006-04-01 & $\mathrm{H}$ & 9.17 & LB & M3III & VIS & OS: M0 \\
\hline V1001 Cen & GSC 08682-01015 & 2006-04-02 & $\mathrm{H}$ & 7.24 & IA & B4 & SB2 & OS: B4IV/V, OB:? \\
\hline V1003 Sco & HD 149711 & 2006-05-04 & $\mathrm{H}$ & 5.83 & ELL & B3IV & VIS & OS: B2.5IV \\
\hline V1011 Cen & GSC 09011-04767 & 2006-09-01 & $\mathrm{F}$ & 8.47 & LB & M5 & $?$ & OS: M4/M5 \\
\hline V1026 Sco & GSC 06199-00618 & 2006-04-02 & $\mathrm{H}$ & 8.85 & IA & F0IV-III & $?$ & OS: A8Ve \\
\hline V1045 Sco & GSC 05624-00995 & 2006-08-11 & $\mathrm{F}$ & 8.08 & LB & K7III & $?$ & OS: K5III \\
\hline V1048 Sco & GSC 07342-00752 & 2006-08-11 & $\mathrm{F}$ & 9.05 & LB & K7 & $?$ & OS: K5III: \\
\hline V1052 Sco & GSC 05613-00357 & 2006-09-13 & $\mathrm{F}$ & 8.62 & LB & M2 & $?$ & OS: K5 \\
\hline V1053 Sco & GSC 05625-00721 & 2006-04-12 & $\mathrm{F}$ & 8.03 & LB: & M4IV-III & $?$ & OS: M4III \\
\hline V1066 Sco & GSC 07358-00675 & 2006-06-11 & $\mathrm{F}$ & 9.08 & $?$ & M2III & $?$ & OS:M \\
\hline V1080 Sco & GSC 07883-00697 & 2006-04-01 & $\mathrm{H}$ & 7.65 & IA & $\mathrm{A} 0 \mathrm{~V}+\mathrm{A} 0 \mathrm{~V}$ & SB2 & OS: B9.5IV, OB: VIS \\
\hline V1085 Sco & GSC 07388-00568 & 2006-05-24 & $\mathrm{F}$ & 9.02 & $?$ & M5III & $?$ & OS: M2 \\
\hline V1434 Aql & GSC 01030-00103 & $2006-08-15$ & $\mathrm{~F}$ & 7.56 & LB & M3III & $?$ & OS: M \\
\hline V1440 Aql & GSC 05140-00148 & 2006-05-18 & $\mathrm{F}$ & 8.4 & ELL & $\mathrm{B} 1 \mathrm{~V}+\mathrm{B} 1 \mathrm{~V}$ & SB2 & OS: B8, OB: VIS \\
\hline V1442 Aql & GSC 01048-00621 & 2006-05-18 & $\mathrm{F}$ & 7.46 & LB & K7III-IV & $?$ & OS: K0 \\
\hline V1443 Aql & GSC 01040-00260 & 2006-04-02 & $\mathrm{H}$ & 8.95 & GCAS & $\mathrm{B} 5 \mathrm{Ve}$ & $?$ & OS: B9V \\
\hline V1450 Aql & GSC 01042-02041 & 2006-04-02 & $\mathrm{H}$ & 8.98 & EB & $\mathrm{A} 0 \mathrm{~V}+\mathrm{A} ?$ & SB2 & OS: A0, OB: Y \\
\hline V1453 Aql & GSC 05143-00240 & 2006-05-12 & $\mathrm{F}$ & 9.1 & LB & MOIV & $?$ & OS: K5 \\
\hline V1459 Aql & GSC 01060-00614 & 2006-08-08 & $\mathrm{F}$ & 8.48 & LB & M3III+M & SB2 & OS: M2, OB:? \\
\hline V1460 Aql & GSC 05728-01515 & 2006-06-13 & $\mathrm{F}$ & 9.23 & $?$ & M2III & ? & OS: K5 \\
\hline V1465 Aql & GSC 00492-01519 & 2006-05-12 & $\mathrm{F}$ & 9.22 & $\mathrm{ACV}$ & $\mathrm{F} 2 \mathrm{~V}$ & $?$ & OS: A5 \\
\hline V1471 Aql & GSC 00498-01575 & 2006-05-04 & $\mathrm{H}$ & 8.42 & EB & $\mathrm{A} 1 \mathrm{~V}+\mathrm{A} 1 \mathrm{~V}$ & SB2 & OS: A0, OB: Y \\
\hline V2300 Oph & GSC 00442-01788 & 2006-05-04 & $\mathrm{H}$ & 6.7 & ELL & $\mathrm{A} 0 \mathrm{~V}$ & $?$ & OS: A0 \\
\hline V2350 Oph & GSC 05047-00021 & 2006-09-13 & $\mathrm{F}$ & 7.41 & LB & M3III & $?$ & OS:M \\
\hline V2354 Oph & GSC 06221-00890 & 2006-09-09 & $\mathrm{F}$ & 8.58 & LB & M2III & $?$ & OS: M2III: \\
\hline V2356 Oph & GSC 00979-00970 & $2006-08-23$ & $\mathrm{~F}$ & 7.07 & LB & G8I-II & $?$ & OS: K5 \\
\hline V2361 Oph & GSC 00410-02537 & 2006-09-01 & $\mathrm{F}$ & 8.62 & LB & M5 & $?$ & OS: M4 \\
\hline V2362 Oph & GSC 00406-02288 & 2006-09-01 & $\mathrm{F}$ & 8.72 & LB & M5III & $?$ & OS:M \\
\hline V2366 Oph & GSC 00399-01658 & 2006-09-13 & $\mathrm{F}$ & 8.97 & LB & M0 & $?$ & OS: K5 \\
\hline V2369 Oph & GSC 00982-02178 & 2006-04-01 & $\mathrm{H}$ & 8.54 & BY & $\mathrm{G} 9+?$ & SB3 & OS: G5, OB: VIS \\
\hline V2370 Oph & GSC 00995-02245 & 2006-09-01 & $\mathrm{F}$ & 9.79 & LB & M0 & $?$ & \\
\hline V2386 Oph & GSC 00428-01318 & 2006-09-01 & $\mathrm{F}$ & 7.41 & LB & M4IV-III & $?$ & OS: M \\
\hline V341 Sge & GSC 01606-01704 & 2006-05-04 & $\mathrm{H}$ & 7.67 & GCAS & $\mathrm{B} 2 \mathrm{Ve}$ & $?$ & OS: B2.5V \\
\hline V344 Hya & GSC 06710-00436 & 2006-04-09 & $\mathrm{F}$ & 7.28 & LB & M2III & $?$ & \\
\hline V344 Peg & GSC 01712-00611 & 2006-08-07 & $\mathrm{F}$ & 9.63 & LB & M7III & $?$ & OS: M5 \\
\hline V349 Vel & GSC 08192-04033 & 2006-04-01 & $\mathrm{H}$ & 9.64 & $\mathrm{ACV}$ & $\mathrm{F} 2+?$ & $\mathrm{Y}$ & OS: F3IVp \\
\hline V350 Nor & GSC 09036-03305 & 2006-04-01 & $\mathrm{H}$ & 9.2 & $*$ & $\mathrm{~B} 5+?$ & SB2 & OS: B8/B9Ib/II, OB: ? \\
\hline V355 Нyа & GSC 06744-00025 & 2006-09-07 & $\mathrm{F}$ & 8.33 & LB & M2III & $?$ & \\
\hline V355 Pav & GSC 09290-00176 & $2006-05-24$ & $\mathrm{~F}$ & 8.37 & $?$ & M2IV-III & $?$ & OS: M2III: \\
\hline V357 Pav & GSC 09291-01224 & 2006-05-04 & $\mathrm{H}$ & 7.94 & $\mathrm{ACV}$ & B9III & $?$ & OS: B8III \\
\hline V357 Vel & GSC 08615-02755 & 2006-06-10 & $\mathrm{F}$ & 8.31 & $?$ & K0IV-III & $?$ & OS: K0III \\
\hline V363 Nor & GSC 08710-01594 & 2006-08-11 & $\mathrm{F}$ & 7.89 & LB & M3III & $?$ & OS: M2/M3III: \\
\hline V369 Pav & GSC 09313-00616 & 2006-05-24 & $\mathrm{F}$ & 8.40 & $?$ & M2III & $?$ & \\
\hline V370 Nor & GSC 08711-01144 & $2006-08-27$ & $\mathrm{~F}$ & 8.17 & LB & $\mathrm{K} 7+?$ & SB2 & OS: K5/M0III:, OB: VIS \\
\hline
\end{tabular}

\subsection{Selected individual cases}

In this section, we give a few examples of different kinds of objects we have encountered. These examples include both "typical cases" and "special cases", in order to give an impression of the wide range of phenomena we have to deal with, and which our automated procedures will have to be able to handle. Subsequent publications will deal in depth with interesting special cases, be devoted to resolving binaries, deal with various classes of stars, and will address the general problem of variability classification.

\subsubsection{V349 Vel - visual and spectroscopic binary}

V349 Vel is a close visual binary, separated by $1.1^{\prime \prime}$. The CCDM (Dommanget \& Nys 1994) lists spectral type F5 for the A component $\left(m_{v}=9.8\right)$, while the B component must be a later type at $m_{v}=11.1$. Our HARPS spectrum, obtained on 2006-04-01, reveals a complex CCF with at least four well defined components, the dominant one being a F2 type. Due to their proximity, the fiber entrance $\left(1.0^{\prime \prime}\right)$ includes light from both visual components. Thus, the A and B components both spilt up into SB2 
Table 2. continued.

\begin{tabular}{|c|c|c|c|c|c|c|c|c|}
\hline \multicolumn{9}{|c|}{ Properties of the stars in our sample } \\
\hline V371 Nor & GSC 08715-01929 & 2006-04-02 & $\mathrm{H}$ & 9.35 & BY & $\mathrm{K} 5 \mathrm{~V}$ & $?$ & OS: K2V \\
\hline V375 Nor & GSC 08316-01234 & 2006-09-13 & $\mathrm{F}$ & 9.79 & LB & K2IV-III & $?$ & OS: K5 \\
\hline V384 Pav & GSC 09112-00897 & 2006-05-12 & $\mathrm{F}$ & 7.99 & LB & M3III & ? & OS: M3/M4III \\
\hline V389 Pav & GSC 09321-01375 & 2006-04-30 & $\mathrm{F}$ & 7.61 & LB & MOIV & $?$ & OS: M1/M2III \\
\hline V390 Pav & GSC 09114-01109 & 2006-05-04 & $\mathrm{H}$ & 9.03 & BY & K3V & VIS & OS: K2Vp \\
\hline V412 Pup & GSC 07659-03359 & 2006-04-09 & $\mathrm{F}$ & 8.74 & LB & M4III & ? & OS: K5 \\
\hline V414 Pup & GSC 05420-00844 & 2006-04-01 & $\mathrm{H}$ & 8.80 & $\mathrm{ACV}$ & $\mathrm{A} 0 \mathrm{I}+\mathrm{A}$ & SB2 & OS: Ap, OB: Y \\
\hline V422 Pup & GSC 07133-02165 & 2006-04-09 & $\mathrm{F}$ & 9.04 & $?$ & M4III & VIS & OS: K2 \\
\hline V424 Pup & GSC 06563-02968 & 2006-04-09 & $\mathrm{F}$ & 8.93 & LB & K7IV-III & ? & OS: K5 \\
\hline V429 Pup & GSC 06000-01200 & 2006-06-06 & $\mathrm{F}$ & 8.90 & $?$ & K5III & $?$ & OS: K5 \\
\hline V432 Pup & GSC 06005-05152 & 2006-04-01 & $\mathrm{H}$ & 6.67 & $\mathrm{ACV}$ & $\mathrm{A} 3 \mathrm{IV}-\mathrm{V}+\mathrm{A} ?$ & SB2 & OS: A1IV, OB:? \\
\hline V435 Pup & GSC 07130-01521 & 2006-04-09 & $\mathrm{F}$ & 8.33 & LB & MOIII & $?$ & OS: K5 \\
\hline V4376 Sgr & GSC 06258-00907 & 2006-04-02 & $\mathrm{H}$ & 9.14 & ELL & F8IV-III & $?$ & OS: F7V \\
\hline V4377 Sgr & GSC 07399-00071 & $2006-05-24$ & $\mathrm{~F}$ & 8.88 & $?$ & K7III & $?$ & OS: K5 \\
\hline V4385 Sgr & GSC 06855-03418 & 2006-05-04 & $\mathrm{H}$ & 7.42 & ELL & $\mathrm{B} 8+\mathrm{F} 2 \mathrm{p}$ & SB2 & OS: A2/A3V+, OB:? \\
\hline V4393 Sgr & GSC 06277-01745 & $2006-05-24$ & $\mathrm{~F}$ & 7.63 & $?$ & $\mathrm{~K} 5+?$ & SB2 & OS: K5/M0III:, OB: ? \\
\hline V4413 Sgr & GSC 06883-00930 & 2006-06-12 & $\mathrm{F}$ & 8.81 & $?$ & M4/5II & $?$ & OS: M2/M3III \\
\hline V4422 Sgr & GSC 06312-00760 & 2006-04-30 & $\mathrm{F}$ & 8.01 & LB & M2IV & $?$ & OS: M2III \\
\hline V4432 Sgr & GSC 07453-00754 & 2006-06-13 & $\mathrm{F}$ & 8.27 & $?$ & M4III & $?$ & OS: M2/M3III \\
\hline V4436 Sgr & GSC 07948-01704 & 2006-06-11 & $\mathrm{F}$ & 8.92 & $?$ & M3III & $?$ & OS: M2III: \\
\hline V4440 Sgr & GSC 07949-00463 & $2006-05-24$ & $\mathrm{~F}$ & 8.74 & $?$ & K5III & $?$ & OS: K5 \\
\hline V494 Car & GSC 09213-01493 & 2006-04-09 & $\mathrm{F}$ & 9.24 & LB & M6III & $?$ & OS: M5 \\
\hline V538 Car & GSC 08967-00342 & 2006-04-21 & $\mathrm{F}$ & 7.64 & LB & M6III-II & $?$ & OS: M3 \\
\hline V715 CrA & GSC 07901-00567 & 2006-05-04 & $\mathrm{H}$ & 6.8 & $\mathrm{ACV}$ & $\mathrm{B} 5+\mathrm{A} 3 ?$ & SB2 & OS: A0II/IIIp, OB: ? \\
\hline V771 Mon & GSC 04847-02925 & 2006-06-06 & $\mathrm{F}$ & 8.14 & $?$ & M2III & VIS & OS: M \\
\hline V830 Ara & GSC 08342-04690 & 2006-04-02 & $\mathrm{H}$ & 8.11 & GCAS & B1III-IIe & $?$ & OS: B2Ib/IIpe \\
\hline V841 Ara & GSC 08720-01374 & 2006-05-04 & $\mathrm{H}$ & 8.73 & BY & K0V & VIS & \\
\hline V844 Ara & GSC 08327-01945 & $2006-08-23$ & $\mathrm{~F}$ & 8.38 & LB & K0IV-III & $?$ & OS: K2/K3III \\
\hline V845 Ara & GSC 08331-01099 & 2006-09-07 & $\mathrm{F}$ & 8.37 & LB & M5 & $?$ & OS: M3/M4III \\
\hline V856 Ara & GSC 08739-02203 & 2006-08-31 & $\mathrm{F}$ & 8.34 & LB & K5III & $?$ & OS: K4III: \\
\hline V857 Ara & GSC 09052-01117 & 2006-04-01 & $\mathrm{H}$ & 9.59 & BY & $\mathrm{K} 2 \mathrm{~V}$ & $?$ & OS: G8/K0V \\
\hline V870 Ara & GSC 08751-01331 & $2006-05-24$ & $\mathrm{~F}$ & 8.92 & $?$ & G0 & SB2 & OS: F8, OB: Y \\
\hline V907 Her & GSC 00961-01721 & 2006-06-11 & $\mathrm{F}$ & 8.31 & $?$ & K7V & $?$ & OS: K5 \\
\hline V915 Her & GSC 01524-00686 & $2006-08-23$ & $\mathrm{~F}$ & 8.32 & LB & M2III & $?$ & OS: M \\
\hline V939 Cen & GSC 07783-00935 & 2006-07-02 & $\mathrm{F}$ & 8.25 & LB & M4III & $?$ & OS: M3III \\
\hline V940 Cen & GSC 08257-01308 & 2006-04-02 & $\mathrm{H}$ & 9.59 & BY & K0III & $?$ & OS: G8/K0V \\
\hline V944 Cen & GSC 07772-01464 & 2006-04-09 & $\mathrm{F}$ & 9.23 & LB & M2III & $?$ & OS: K8 \\
\hline V974 Cen & GSC 07269-00319 & 2006-04-01 & $\mathrm{H}$ & 7.67 & ELL & $\mathrm{F} 2 \mathrm{~V}+\mathrm{F} ?$ & SB2 & OS: F2, OB: VIS \\
\hline V976 Cen & GSC 08999-00906 & 2006-04-01 & $\mathrm{H}$ & 9.38 & $\mathrm{ACV}$ & FOIII-II & $?$ & OS: A7IV/V \\
\hline V981 Her & GSC 01554-01928 & 2006-08-15 & $\mathrm{F}$ & 7.24 & LB & M2III & $?$ & OS: M \\
\hline V982 Cen & GSC 07794-00587 & 2006-04-02 & $\mathrm{H}$ & 9.6 & BY & $\mathrm{K} 4 \mathrm{~V}+\mathrm{K}$ & SB2 & OS: K2V, OB: VIS \\
\hline V988 Cen & GSC $07280-01160$ & 2006-04-02 & $\mathrm{H}$ & 9.77 & BY & $\mathrm{K} 0+\mathrm{K}+?$ & SB3 & OS: K0V, OB:? \\
\hline V999 Her & GSC 01574-01910 & 2006-06-12 & $\mathrm{F}$ & 8.90 & $?$ & M2 & $?$ & OS: M0 \\
\hline VX PsA & GSC 07492-00927 & 2006-07-01 & $\mathrm{F}$ & 6.97 & LB & M3III & $?$ & \\
\hline WY PsA & GSC 07511-00060 & 2006-08-09 & $\mathrm{F}$ & 8.71 & LB & M3III & $?$ & OS: M2III \\
\hline XZ Pyx & GSC 06569-02459 & 2006-04-09 & $\mathrm{F}$ & 9.22 & LB & M3IV-III & $?$ & OS: M \\
\hline ZZ Рyx & GSC 07139-01937 & 2006-04-18 & $\mathrm{F}$ & 8.63 & LB & $\mathrm{K} 3 \mathrm{~V}$ & $?$ & OS: K2 \\
\hline
\end{tabular}

${ }^{6}$ Hereafeter OS. ${ }^{7}$ Hereafter OB. ${ }^{8}$ Hereafter OV.

spectroscopic binaries. Based on the line strengths and the rotational broadening, which is expected to be higher for $\mathrm{F}$ type stars than for later spectral types, we identify the lines of each of the four components as shown in Fig. 1. While new epoch spectroscopy would be needed to determine the orbital parameters, we consider it beyond doubt that V349 Vel is a four component multiple system composed of two SB2 binaries orbiting each other

The GCVS variability type is $\alpha^{2}$ Canum Venaticorum $(\mathrm{ACV})$, which is reserved for magnetic B-A stars with peculiar spectra. We suspect that the SB4 appearance of V349 Vel may have been misinterpreted as spectral peculiarities. We do not see evidence of magnetic activity in the spectrum, hence we also consider BY Dra type variability unlikely. From our spectral analysis (Sect. 4.2) we find that the parameters of the primary component is consistent with an early $\mathrm{F}$ type star near the main sequence. Thus, the primary component of V349 Vel could well belong to the $\delta$ Scuti or $\gamma$ Dor variables. Alternatively, one or both of the SB2 components could show eclipses. Otero (2003) listed V349 Vel as an EA-type eclipsing binary, with $P=3.02 \mathrm{~d}$, and notes that additional shorter periods may be present as well. More detailed photometric or spectroscopic 


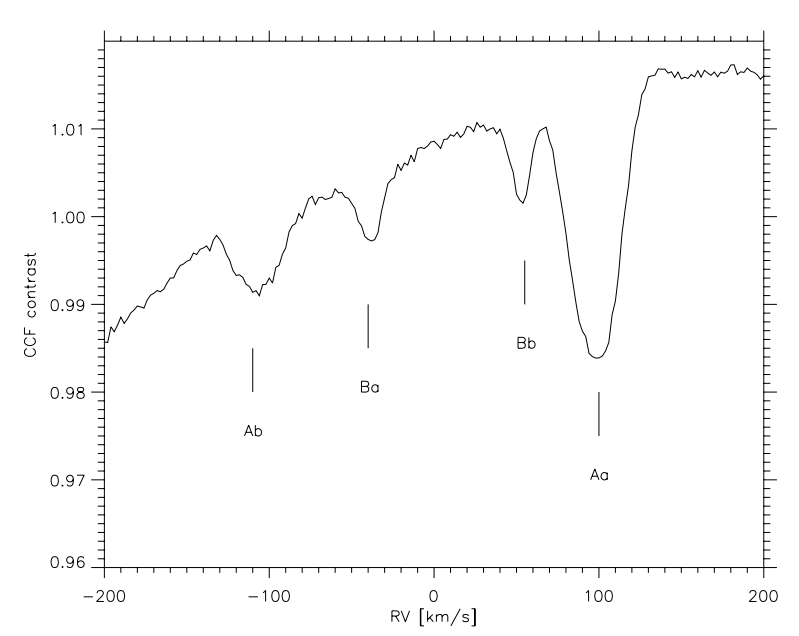

Fig. 1. The CCF of V349 Vel. Capitals denote the CCDM designation, while lower case letters label the individual components of each visual component.

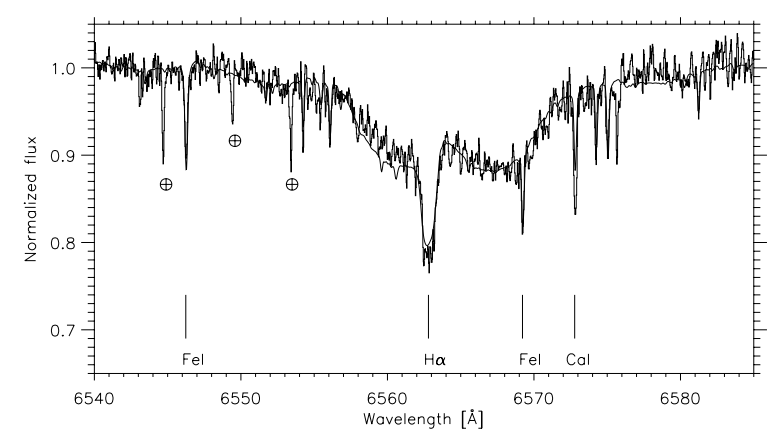

Fig. 2. $\mathrm{H} \alpha$ line region of BC Gru. Over-plotted is the best fitting synthetic spectrum (see text). The marked lines all belong to the tertiary component, except the telluric lines marked $\oplus$.

observations are needed to understand the components of the system better.

\subsubsection{BC Gru - a triplet eclipsing binary}

BC Gru is an very short period $(P=0.2662 \mathrm{~d})$ eclipsing contact binary, listed as a late-type contact $\mathrm{W}$ system in the catalog by Malkov et al. (2006). Apparently, this object has not even been observed with low-resolution or objective prism surveys.

Our FEROS spectrum of BC Gru not only confirms the contact binary nature, but also reveals a third component, as evident in Fig. 2. All three stars have approximately the same spectral type. We have performed a simple spectral fitting using archive HARPS spectra of the K2V star HD 22049 ( $\epsilon$ Eri). We have artificially broadened and Doppler-shifted two copies of this template, then combined with an unbroadened copy of the same template to emulate the spectrum of BC Gru. The fitting has been done using STARMOD (Barden 1985; Montes et al. 1995, 2000). A better fit could likely be obtained using different templates for each component, but in order to do a more accurate analysis, one would need spectra at several different orbital phases. Our analysis yields rotational velocities of $(v \sin i)_{a}=165 \pm 50 \mathrm{~km} \mathrm{~s}^{-1}$ and $(v \sin i)_{b}=142 \pm 50 \mathrm{~km} \mathrm{~s}^{-1}$, while the sharp-lined component is a very slow rotator, with measured $(v \sin i)_{c}=6 \pm 3 \mathrm{~km} \mathrm{~s}^{-1}$. In Fig. 2 the $a$ component is the red-most one.

\subsubsection{V4385 Sgr - composite spectrum}

V4385 Sgr is a known composite spectrum star, classified as an ellipsoidal variable, and hence a close early-type binary system with geometrically distorted components (Kazarovets et al. 1999a). Its variability was detected by Hipparcos, which found a period of $2.62 \mathrm{~d}$ with peak-to-peak variations of $0.08 \mathrm{mag}$. It was observed during lunar occultations (Dunham 1974; Schmidtke et al. 1989) and recorded as double in the University of Texas Special Double Star list (Schmidtke 1979). Previous prism observations (typically 39/91 $\AA \mathrm{mm}^{-1}$ ) were obtained by several authors (see, e.g., Houk \& Cowley 1975; Reed \& Beatty 1995; Garrison et al. 1977; Abt et al. 1979; Kennedy 1983; Kawabata et al. 2000). The composite spectrum is usually classified combining an early B to late A star component, plus an early F. In particular, Garrison et al. (1977) classified V 4385 Sgr as a shell star having Mg, He, and Si like a B5 star but with very broad $\mathrm{H}$ and $\mathrm{Ca}$ II and the rest of the spectrum like an F2 star. SIMBAD however, classifies it as a $\mathrm{A} 2 / \mathrm{A} 3 \mathrm{~V}$ primary and unknown secondary, which is clearly incorrect.

Our high quality VSOP spectrum of V4385 Sgr $(S / N=130$ at $550 \mathrm{~nm}$ ) was obtained on 2006-05-04 using HARPS, and is the first full optical range high resolution spectrum of the source. The HARPS CCF is single-peaked at high contrast (11\%) but asymmetric, as expected due to geometric distortion. The CCF is constructed using a G2 mask, and hence represents the average line profile of the F-component only at a radial velocity of $14.5 \mathrm{~km} \mathrm{~s}^{-1}$. The $F W H M$ of the CCF is $13 \mathrm{~km} \mathrm{~s}^{-1}$, which is a safe upper limit for $v \sin i$. Thus the rotation is significantly slower than for the average early F-star, which is surprising given the short variability period, and the fact that binary evolution tends to increase rotation rates as the orbit shrinks.

The spectrum (Fig. 3) shows the typical He I lines of the B component superimposed on the metallic-line spectrum. Note that the RV of the two components seem compatible within the uncertainties.

The VSOP spectrum shows Balmer emission lines, strongest at $\mathrm{H} \alpha\left(F W H M=132 \mathrm{~km} \mathrm{~s}^{-1}\right)$, core fill-in at $\mathrm{H} \beta$ and $\mathrm{H} \gamma$ (see also Merrill \& Burwell 1950). Weak P Cyg profiles can be seen in several lines, however not in the Balmer lines. Note that the lines of both components, including the strong $\mathrm{H} \alpha$ emission, are compatible with $R V=14.5 \mathrm{~km} \mathrm{~s}^{-1}$. The interstellar Na doublet is saturated and presents also a P Cyg profile from the underlying stellar spectrum.

Based on the observed emission properties, and on the undisputable two-component nature, we can safely rule out that the $2.62 \mathrm{~d}$ period can be due to $\gamma$-Dor like pulsations in a single F star. Rather, due to the slow rotation of the F-star and the apparent same RV of the two components, we propose that V4385 Sgr could be a near pole-on viewed close binary system, showing only slight eclipses. We propose that the orbital period is equal to the photometric period, caused either by a partial eclipse of the smaller component, or by variations in the wind structure over the orbit. Unfortunately, only scattered photometric data exists, as summarized by Reed (1998), making it difficult at this point to test the hypothesis. Given its brightness, the star would be an easy target for small telescopes.

\subsubsection{V1045 Sco - strong lithium absorption}

V1045 Sco (HD 144377, $V=8.06$ ) is classified as K5III in SIMBAD, with only one bibliographic reference (Kazarovets et al. 1999b). It is an IRAS source with a flux of $2 \mathrm{Jy}$ in the $12 \mu \mathrm{m}$ channel, and decreasing fluxes towards the longer wavelength 


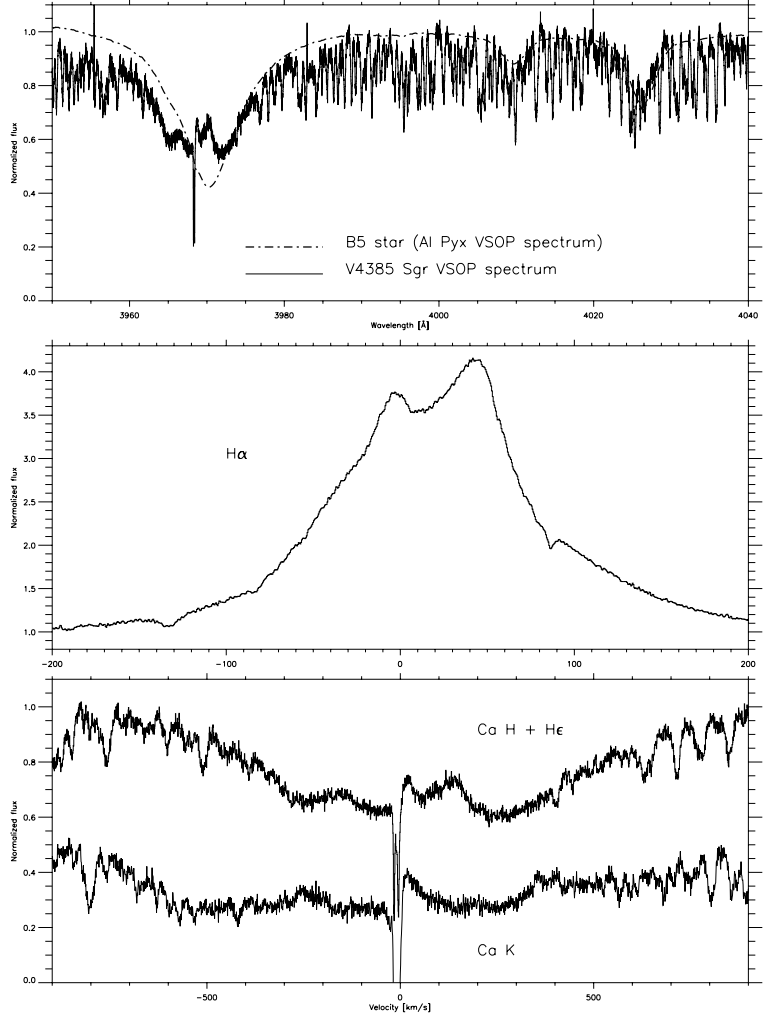

Fig. 3. Plot of selected spectral regions of V4385 Sgr. Fluxes are normalized to the continuum. Top: over-plotted is the VSOP spectrum of the B5 star AI Pyx. The composite B+F nature of the V4385 Sgr spectrum is evident. Middle: $\mathrm{H} \alpha$ profile. Bottom: $\mathrm{Ca}$ II $\mathrm{H}+\mathrm{K}$ lines. The $\mathrm{K}$ line has been shifted down for display purposes. Note that B stars do not show these lines, so the complex structure (also visible in top panel) comes from the F-star alone.

bands. The mid-IR flux is therefore well above the expected photospheric level, and indicates circumstellar, dusty, material.

We have secured two FEROS spectra of this object, on 2006-08-11 and 2006-09-16. Our spectral typing procedure (Sect. 4.2) yields consistently a somewhat later spectral type, $\mathrm{K} 7$, compatible with a giant (or sub-giant). A radial velocity of $25.77 \mathrm{~km} \mathrm{~s}^{-1}$ is determined. A striking feature is the $\mathrm{H} \alpha$ profile (Fig. 4). The line is in absorption, but exhibits slightly asymmetric double-peaked emission features in both wings, possibly indicative of a rotating disk.

The star exhibits a strong lithium (6708 $)$ ) absorption, with a measured equivalent width of $0.48 \AA$. Taken together with its late spectral type, this is a strong youth indicator. Note that the star is compatible with being a (weak-lined, as $\mathrm{H} \alpha$ emission is inversed) $\mathrm{T}$ Tauri star according to the commonly accepted spectroscopic criteria by Martin (1997). Comparing its lithium strength to recent measurements in a sample of nearby, young objects (Torres et al. 2006), we conclude that it is at least as young as the $\approx 10$ Myr old $\beta$ Pictoris moving group members. Its apparent youth and its location in the northern outskirts of the Upper Scorpius OB association indicates that V1045 Sco might be related to, or in the foreground of, this 5 Myr young association (Preibisch \& Zinnecker 1999). This is supported by the apparent presence of remnant circumstellar material, inferred by the $\mathrm{H} \alpha$ profile and mid-IR excess. Its measured heliocentric $R V=25.77 \mathrm{~km} \mathrm{~s}^{-1}$ is, however, inconsistent with the bulk motion of Upper Scorpius members that peak around $-10 \mathrm{~km} \mathrm{~s}^{-1}$ (see, e.g., Sartori et al. 2003; Jilinski et al. 2006) The star may

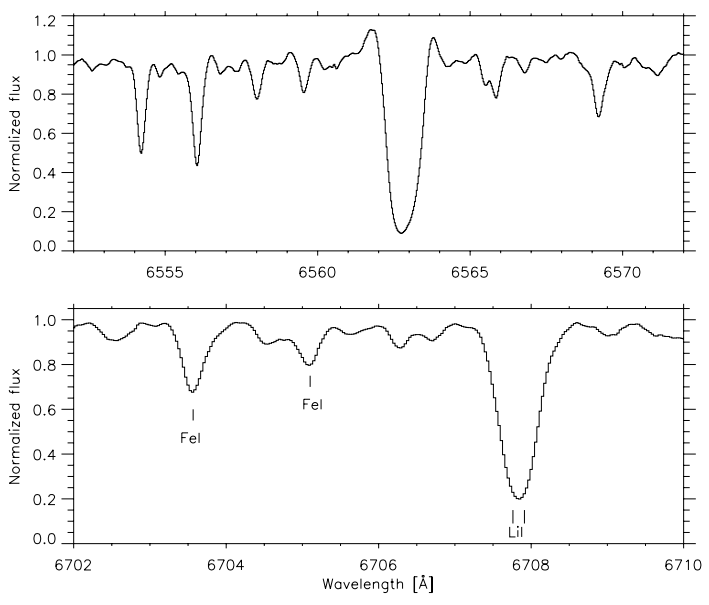

Fig. 4. Selected spectral regions of V1045 Sco: Top: $\mathrm{H} \alpha$ profile. Note the emission on both the red and blue edges, and the strong reversal. Bottom: the lithium line region.

therefore well be located in the foreground of the association, possibly related to the Gould Belt (Guillout et al. 1998).

\subsubsection{DM Boo - reclassification}

DM Boo (HD 120447, HIP 67499, BD+11 2604) has been classified as an irregular variable star of spectral type G5 (Simbad) and a Johnson $V$ magnitude and $(B-V)$ color of 8.68 and 0.67 respectively. The only photometric monitoring found in the literature is the one carried out by the Northern Sky Variability Survey (NSVS; Woźniak et al. 2004). The average brightness of the star in their no-filter observations (ROTSE) gives a value of $8.59 \mathrm{mag}$, with a scatter of less than $0.08 \mathrm{mag}$ (and precision of $0.01 \mathrm{mag}$ ). Hipparcos gives a parallax for the star of $5.80 \pm 1.25$ mas, corresponding to a distance of $d=172 \mathrm{pc}$.

There are also $u v b y$ observations (one set, from 1993) and the $\mathrm{V}$ and $\mathrm{B}$ values from Tycho. The Tycho-2 Spectral Type Catalog (Wright et al. 2003) converts the G5 spectral class into a temperature of $T_{\text {eff }}=5150 \mathrm{~K}$. The GCVS lists the star as a IB type, i.e., a poorly studied irregular variable of intermediate to late spectral type.

The VSOP FEROS spectrum was taken on 2006-08-12 with an integration time of $600 \mathrm{~s}$. From the CCF we derive a $v \sin i$ of $\approx 65 \mathrm{~km} \mathrm{~s}^{-1}$ and a radial velocity of $-42 \mathrm{~km} \mathrm{~s}^{-1}$. The most prominent features are strong $\mathrm{Ca}$ II $\mathrm{H} \& \mathrm{~K}$ emission cores, which, together with the fast rotation, points to a young active star. Analysis of the lithium line region reveals higher than solar photospheric lithium abundance, confirming the notion of a relatively young, active star. We thus feel confident classifying DM Boo as a BY Draconis star, noting the low photometric amplitude typical of such stars.

\subsection{Evaluation in terms of spectral type and binarity}

To compute some statistics of the VSOP observations, we must rely on "old" values as they were before VSOP observations. This concerns mostly the spectral type and the binary status. The old spectral type is determined automatically by querying SIMBAD. To determine the binary status of a target, we query VizieR catalogues with the Multiple and Double stars, Spectroscopic, Cataclysmic and Eclipsing binary keywords to see if the star belongs to one of these classes. If the star belongs to a catalogue of spectroscopic binaries, or one providing orbits 
of stars, the star is said to be a binary. If the star belongs to some visual binaries or multiple and double stars catalogs, the star is suspected to be a visual binary. Finally, if it only belongs to the Tycho's and Hipparcos catalogues, the suspected binary status is inferred from the MultFlag parameter of the catalogue. The procedure is quite complex, and is described in the wiki website (Binary Status page). We have tested various combination of criteria, which seems to converge to a relatively stable results. Our statistics are based on these tests.

The main points of the VSOP observations can be summarized as follows:

- VSOP observations allow to revise more than two third of the spectral types;

- among these revisions about $4 \%$ had no previous spectral type, about $25 \%$ of the revisions implies a complete change of spectral class (say from $\mathrm{F}$ to $\mathrm{G}$ ), about $30 \%$ are changes by more than one subclass (say from F3 to F5), and about $20 \%$ are changes of one subclass. The remaining approximately $20 \%$ are assignment or re-assignment of luminosity class.

- there are no clear trends or systematic biases toward revisions of any given spectral class, spectral subtype, or luminosity class.

- 40 of our targets (about 20\%) appear to be spectroscopic binaries. Of these, 22 are previously unknown SB2 systems, while we have identified four new SB3 systems.

\section{The future of VSOP}

This paper has presented the first data release from VSOP, covering ESO Period 77. Observations are ongoing in Periods 78 and 79 , and data from these periods will be released as soon as the periods end.

\subsection{Next steps}

Future space missions like COROT and GAIA will provide a wealth of data for variable stars that will be observed with unprecedented precision and sampling of their light curves. Nevertheless, situations of ambiguity in the determination of the variability type can arise. Also, as in the case of Hipparcos, it is expected that these data will lead to the discovery of a number of new classes of variables. For all of them, it will be necessary to refine their variability type and position in the HR diagram with spectroscopic data. This is what VSOP has been doing with objects of the GCVS, but the sheer number of stars provided by these missions would deem this classification impossible to be determined by hand.

For the next steps, the main aim of VSOP is to acquire and develop the necessary tools to provide good and reliable spectral and variability classification of stars automatically from the available data, either the spectrum, the light curve, color information, or combinations of these.

\subsection{Automatic variability classification}

There have been in the past several attempts to mine large archives of variable objects data, mainly based on photometric time series and, sometimes, on photometric colours as well. Good examples in the field of classification, are the series of papers on MACHO, OGLE and ASAS data (for RR Lyrae stars, for example, see Alcock et al. (2003), Soszynski et al. (2003) and Wils et al. (2006), for MACHO, OGLE and the NSVS respectively), where catalogues of distinct variability classes are compiled according to several selection criteria (rules) and, in some occasions, with human intervention. Basically, the problem of supervised classification of variable objects can be described as that of defining general boundaries (hard or fuzzy) in the hyperspace of the features that describe the classes, based on a set of examples of each class.

The field of Machine Learning and Pattern Recognition offers a wealth of alternatives for defining more complex, flexible, and general boundaries than the hyperboxes used in the compilation of catalogues, minimizing at the same time the human intervention in the classification process. In this sense, the VSOP automatic variability classifier will build upon a previous effort (Sarro et al. 2006; Debosscher et al. 2006, 2007) carried out during the past few years to i) create a well defined training set of bona fide variable objects belonging to the most important and numerous classes, ii) analyse the most relevant and informative features that describe these classes and iii) to study and compare different approaches to the task of classification, from Bayesian Networks (Pearl 1988) to Bayesian averages of artificial neural networks (Neal 1996) or SVMs (Support Vector Machines, Vapnik 1995). The development of this classifier was motivated by the wealth of data expected from the COROT space mission (Baglin et al. 2000) and was thus designed to facilitate the indepth analysis of representative samples of these classes as observed by COROT. It produces probabilistic class assignements based on photometric time series parameters (harmonic amplitudes of component frequencies, phase differences, amplitude ratios, etc.). The objective is twofold: i) to generate class specific object lists for further analysis by COROT's Additional Program scientists and ii) to detect objects lying outside the known density distribution of objects/classes in the parameter space; these objects can possibly represent new astrophysical scenarios for variability. The classifier presented in Debosscher et al. (2007) has now been extended by the same authors to incorporate the photometric colours $B-V, V-I, J-H, H-K$ and, for a reduced number of classes, also Strömgren indices (Sarro et al. 2007).

The effort described in the previous paragraphs is now being continued and adapted as part of the GAIA Data Processing and Analysis Consortium (Gilmore et al. 2000) to incorporate attributes that will be provided by GAIA instruments such as Blue and Red spectrophotometry or spectroscopy near the Calcium infrared triplet (Eyer 2006).

The VSOP automatic classifier is designed as a Virtual Observatory compliant service capable of producing probabilistic class assignements for objects with a wide variety of attributes available (from time series photometry to multiwavelength spectra or photometric colours) and will thus represent the culmination of all the efforts on which it is based.

The development plan necessarily includes a first stage where spectra of at least a representative sample of the COROT training set objects have to be obtained in order to allow for the incorporation of spectral information to the classifier. Here is where VSOP initially will play a major part by collecting this dataset. Subsequent to this, a study will be conducted in order to determine an optimal subset of features providing the best classification performance. Obvious candidate features will be line or band fluxes, equivalent widths, ratios, and/or combinations thereof, line asymmetry measures, and derived physical parameters $\left(T_{\text {eff }}, \log g,[\mathrm{Fe} / \mathrm{H}]\right)$. All these will be subject to the statistical feature analysis classical in Machine Learning applications. 
One obvious requisite of the classifier will be its capability for prediction based on incomplete data. This can happen if the spectral information only covers a fraction of the wavelength range used for training or if the resolution is too poor to separate several lines. Based on these specifications, several state-of-theart machine learning algorithms will be applied to the training set and the performance assessed according to standard figures of merit like overall misclassification rates or the area under the receiver operating curve (Fawcett 2003).

While these efforts are already undergoing in the context of the space missions, the VSOP team is actively taking part in these new developments towards complete automatic classification.

\subsection{Long-term vision}

While it is growing rapidly, VSOP is flexible enough to allow us to imagine a long-term future. VSOP is a project implemented only by astronomers, using the MediaWiki software. With a simple, though large, collection of scripts developed over the year of operation, in addition to the already implemented spectrograph's pipelines, VSOP is a quasi-automatic spectrum production machine whose results are automatically available through a wiki website. As emphasized before, the spectral and variability analysis will also become automatic in the future. The wiki provides a scriptable and clean interface which requires minimum human intervention, while centralizing all the work done by the team. In this sense, it combines both the advantages of an automatically generated content website (homogeneity, reliability, cleanness), with the total flexibility for the contributors to customize a specific point, and for the public to have access to the data, the information and the history. In that perspective, we could imagine an all-automatic wiki-database accepting pipeline-reduced spectra from any observatories in the world, not necessarily dedicated to variable stars.

This larger vision of complete automation while retaining absolute flexibility, is at the core of the VSOP future. As a first step in this direction, we have included the released data in Wikimbad (http://wikimbad.org) as well as directly on our own VSOP server.

\subsection{An open community}

We would like to stress that VSOP is an open community of scientists. We obtain, process, publish, and provide data at a growing rate, and to be able to keep up with that, we welcome any scientist from any background, who wants to contribute to the growth of the project. By joining the VSOP Team, first access rights to the data is granted, which has already spurred several follow-up projects, and will continue to do so. A major commitment of VSOP is the fast release of data, including fully calibrated reduced spectra. Once released, the data will of course be available to the entire community.

We believe in open sharing of information on all levels, and we believe this can be accomplished without sacrificing individual scientific ambitions by basing our collaboration on teamwork and the drive for fast scientific turnover. We would like to conclude with an open invitation to participate in VSOP and its mission, either as part of the VSOP team, or independently through the freely accessible data releases.

Acknowledgements. This research has made extensive use of the Simbad and VizieR databases, and their XML interface, operated at CDS, Strasbourg, Fance. We are very grateful to O. Hainaut for suggesting the project name, and to the referee for constructive comments that improved the paper. M.F. was supported by the Spanish grants AYA2004-05395 and AYA2004-21521-E. Supported by the Gemini Observatory, which is operated by the Association of Universities for Research in Astronomy, Inc., on behalf of the international Gemini partnership of Argentina, Australia, Brazil, Canada, Chile, the UK, and the United States of America.

\section{References}

Abt, H. A., Brodzik, D., \& Schaefer, B. 1979, PASP, 91, 176

Alcock, C., Alves, D. R., Becker, A., et al. 2003, ApJ, 598, 597

Allende Prieto, C. 2003, MNRAS, 339, 1111

Allende Prieto, C., Hubeny, I., \& Lambert, D. L. 2003a, ApJ, 591, 1192

Allende Prieto, C., Lambert, D. L., Hubeny, I., \& Lanz, T. 2003b, ApJS, 147, 363

Asplund, M., Grevesse, N., \& Sauval, A. J. 2005, in Cosmic Abundances as Records of Stellar Evolution and Nucleosynthesis, ed. T. G. Barnes, III \& F. N. Bash, ASP Conf. Ser., 336, 25

Baglin, A., Vauclair, G., \& The COROT Team. 2000, J. A\&A, 21, 319

Baranne, A., Queloz, D., Mayor, M., et al. 1996, A\&AS, 119, 373

Barden, S. C. 1985, ApJ, 295, 162

Bruntt, H., Bikmaev, I. F., Catala, C., et al. 2004, A\&A, 425, 683

Dall, T. H. 2005, Informational Bulletin on Variable Stars, 5617, 1

Dall, T. H., Schmidtobreick, L., Santos, N. C., \& Israelian, G. 2005, A\&A, 438, 317

Dall, T. H., Santos, N. C., Arentoft, T., Bedding, T. R., \& Kjeldsen, H. 2006, A\&A, 454, 341

de Cat, P., Eyer, L., Cuypers, J., et al. 2006, A\&A, 449, 281

Debosscher, J., Aerts, C., \& Vandenbussche, B. 2006, in Astrophysics of Variable Stars, ed. C. Sterken, \& C. Aerts, ASP Conf. Ser., 349, 219

Debosscher, J., Sarro, L., Aerts, C., et al. 2007, A\&A, submitted

Dommanget, J., \& Nys, O. 1994, Communications de l'Observatoire Royal de Belgique, 115, 1

Dunham, D. W. 1974, Occultation Newsletter, 1, 5

Eyer, L. 2006, in 349, Astrophysics of Variable Stars, ed. C. Sterken, \& C. Aerts, ASP Conf. Ser., 15

Fawcett, T. 2003, ROC Graphs: Notes and Practical Considerations for Data Mining Researchers

Galland, F., Lagrange, A.-M., Udry, S., et al. 2005, A\&A, 443, 337

Garrison, R. F., Hiltner, W. A., \& Schild, R. E. 1977, ApJS, 35, 111

Gilmore, G. F., de Boer, K. S., Favata, F., et al. 2000, in p. 453-472, UV, Optical, and IR Space Telescopes and Instruments, ed. J. B. Breckinridge, \& P. Jakobsen, Proc. SPIE, 4013, 453

Guillout, P., Sterzik, M. F., Schmitt, J. H. M. M., Motch, C., \& Neuhaeuser, R. 1998, A\&A, 337, 113

Heiter, U., Kupka, F., van't Veer-Menneret, C., et al. 2002, A\&A, 392, 619

Horne, K. 1986, PASP, 98, 609

Houk, N., \& Cowley, A. P. 1975, University of Michigan Catalogue of TwoDimensional Spectral Types for the HD Stars (Ann Arbor: Univ. Michigan)

Jilinski, E., Daflon, S., Cunha, K., \& de La Reza, R. 2006, A\&A, 448, 1001

Kaufer, A., Stahl, O., Tubbesing, S., et al. 1999, The Messenger, 95, 8

Kawabata, K. S., Hirata, R., Ikeda, Y., et al. 2000, ApJ, 540, 429

Kazarovets, A. V., Samus, N. N., Durlevich, O. V., et al. 1999a, Inform. Bull. Var. Stars, 4659, 1

Kazarovets, A. V., Samus, N. N., Durlevich, O. V., et al. 1999b, Inform. Bull. Var. Stars, 4659, 1

Kazarovets, E. V., Kireeva, N. N., Samus, N. N., \& Durlevich, O. V. 2003, Inform. Bull. Var. Stars, 5422, 1

Kazarovets, E. V., Samus, N. N., Durlevich, O. V., Kireeva, N. N., \& Pastukhova, E. N. 2006, Inform. Bull. Var. Stars, 5721, 1

Keenan, P. C., \& McNeil, R. C. 1976, An atlas of spectra of the cooler stars: Types G, K, M, S, and C. Part 1: Introduction and tables (Columbus: Ohio State University Press, 1976)

Kennedy, P. M. 1983, MK Classification Catalogue Extension (Weston Creek: Mt. Stromlo Obs.)

Kholopov, P. N., Samus, N. N., Frolov, M. S., et al. 1998, in Combined General Catalogue of Variable Stars, 4.1 Ed (II/214A). (1998)

Kurtz, D. W., Elkin, V. G., \& Mathys, G. 2006, MNRAS, 370, 1274

Kurucz, R. 1993, ATLAS9 Stellar Atmosphere Programs and $2 \mathrm{~km} \mathrm{~s}^{-1}$ grid. Kurucz CD-ROM No. 13. Cambridge, Mass.: Smithsonian Astrophysical Observatory

Kurucz, R. L. 2006, in EAS Pub. Ser., ed. P. Stee, 129

Lefever, K., Puls, J., \& Aerts, C. 2007, A\&A, 463, 1093

Malkov, O. Y., Oblak, E., Snegireva, E. A., \& Torra, J. 2006, A\&A, 446, 785

Martin, E. L. 1997, A\&A, 321, 492

Mayor, M., Pepe, F., Queloz, D., et al. 2003, The Messenger, 114, 20

Merrill, P. W., \& Burwell, C. G. 1950, ApJ, 112, 72 
Montes, D., de Castro, E., Fernandez-Figueroa, M. J., \& Cornide, M. 1995, A\&AS, 114, 287

Montes, D., Fernández-Figueroa, M. J., De Castro, E., et al. 2000, A\&AS, 146, 103

Morgan, W. W., Abt, H. A., \& Tapscott, J. W. 1978, Revised MK Spectral Atlas for stars earlier than the sun (Williams Bay: Yerkes Observatory, and Tucson: Kitt Peak National Observatory, 1978)

Neal, R. M. 1996, Bayesian Learning for Neural Networks (New York: Lecture Notes in Statistics Springer Verlag)

Newberg, H. J., \& Sloan Digital Sky Survey Collaboration. 2003, BAAS, 1385

Otero, S. A. 2003, Inform. Bull. Var. Stars, 5480, 1

Pace, G., Pasquini, L., \& Ortolani, S. 2003, A\&A, 401, 997

Pearl, J. 1988, Probabilistic reasoning in intelligent systems: networks of plausible inference (San Francisco, CA, USA: Morgan Kaufmann Publishers Inc.)

Preibisch, T., \& Zinnecker, H. 1999, AJ, 117, 2381

Reed, B. C. 1998 , ApJS, 115, 271

Reed, B. C., \& Beatty, A. E. 1995, ApJS, 97, 189

Rockosi, C. M. 2006, in Am. Astron. Soc. Meet. Abst., 172.04

Sarro, L. M., Debosscher, J., Aerts, C., Garrido, R., Solano, E., \& Vandenbussche, B. 2006, in The CoRoT Mission, ed. M. Fridlund, A. Baglin, J. Lochard, \& L. Conroy, ESA Publications Division, ESA Spec. Publ. 1306, 385
Sarro, L., Debosscher, J., López, M., \& Aerts, C. 2007, A\&A, in preparation Sartori, M. J., Lépine, J. R. D., \& Dias, W. S. 2003, A\&A, 404, 913

Schmidtke, P. C. 1979, PASP, 91, 674

Schmidtke, P. C., Africano, J. L., \& Quigley, R. 1989, AJ, 97, 909

Schmidtobreick, L., Tappert, C., Horst, H., Saviane, I., \& Lidman, C. 2007, A\&A, 461, 943

Selam, S. O. 2004, A\&A, 416, 1097

Solano, E., Catala, C., Garrido, R., et al. 2005, AJ, 129, 547

Soszynski, I., Udalski, A., Szymanski, M., et al. 2003, Acta Astron., 53, 93

Steinmetz, M., Zwitter, T., Siebert, A., et al. 2006, AJ, 132, 1645

Telting, J. H., Schrijvers, C., Ilyin, I. V., et al. 2006, A\&A, 452, 945

Torres, C. A. O., Quast, G. R., da Silva, L., et al. 2006, ArXiv Astrophysics e-prints

Vapnik, V. N. 1995, The nature of statistical learning theory (New York, NY, USA: Springer-Verlag New York, Inc.)

Vogt, N. 2006, A\&A, 452, 985

Wils, P., Lloyd, C., \& Bernhard, K. 2006, MNRAS, 368, 1757

Wilson, O. C., \& Vainu Bappu, M. K. 1957, ApJ, 125, 661

Woźniak, P. R., Vestrand, W. T., Akerlof, C. W., et al. 2004, AJ, 127, 2436

Wright, C. O., Egan, M. P., Kraemer, K. E., \& Price, S. D. 2003, AJ, 125, 359

York, D. G., Adelman, J., Anderson, Jr., J. E., et al. 2000, AJ, 120, 1579 